\title{
Cosmic-Ray Experiments at High Altitudes over a Wide Range of Latitudes
}

\author{
A. T. Biehl, H. V. Neher, and W. C. Roesch \\ California Institute of Technology, Pasadena, California
}

(Received June 20, 1949)

\begin{abstract}
A number of experiments made in a B-29 on the properties of cosmic rays are herein reported. These include the latitude effect of the following, performed along the 80th geographic west longitude from $64^{\circ}$ geomagnetic north to the geomagnetic equator, all at a constant pressure altitude of $30,000 \mathrm{ft}$. ( $3.10 \mathrm{~m}$ of water equivalent): (1) Intensity at the vertical without and with lead absorber; (2) intensity at $45^{\circ}$ West and $45^{\circ}$ East both without and with lead absorber; (3) total ionization intensity; (4) extensive showers. Also measured at $48^{\circ}$ North and at the geomagnetic equator were the following: (1) Zenith angle effect both without and with lead absorber; (2) intensity vs. air pressure without and with lead; (3) extensive showers $v s$. air pressure; (4) local showers. In addition to the above the variation in azimuth of the cosmicray intensity was measured with the various telescopes tipped at $22 \frac{1}{2}^{\circ}, 45^{\circ}$, and $67 \frac{1}{2}^{\circ}$ to the vertical at an elevation corresponding to $2.35 \mathrm{~m}$ of water equivalent at the geomagnetic equator. Two lead thicknesses were used, 10 and $20 \mathrm{~cm}$, inserted between the counter trays. These experiments throw new light on the nature of the primary radiation and on the interaction of the primary particles with the matter through which they pass.
\end{abstract}

\section{INTRODUCTION}

$I^{\mathrm{T}}$ has been found that the intensity of cosmic rays changes with time, especially at high altitudes. ${ }^{1,2}$ The data taken by the Carnegie Institution of Washington ${ }^{3}$ show that changes at or near sea level are world wide and may amount to several percent in a period of one or two months. The percentage fluctuation at high altitudes appears to be considerably greater than at sea level so that cosmic-ray data taken with balloons at one time should not be compared with those taken at another time unless such fluctuations are taken into account.

Since 1935 the group at the California Institute of Technology has made balloon flights with ionization chambers and counter telescopes in Canada, the United States, Mexico and India, measuring the ionization or counting rate as a function of depth below the top of the atmosphere. It is evident that if one point on each of the curves so obtained were simultaneously determined, the curves could be adjusted slightly and then compared. Ideally this one point should be as high on the curves as possible. For the case of no absorber, using a counter telescope, this would be at about $45,000 \mathrm{ft}$. where the peaks of the altitude curves occur. However at heights of $30,000 \mathrm{ft}$., readily reached with B-29's over great distances, the radiation near the vertical is already from one-half to two-thirds its value at the maximum. The present plan was then to fly at a constant barometric pressure to the equator covering the same latitude range or more than had been covered in previous years with balloon instruments.

Although data taken in a flight such as this at 30,000 ft. going north or south are not taken simultaneously

\footnotetext{
${ }^{1}$ Millikan, Neher, and Pickering, Phys. Rev. 66, 295 (1944).

${ }^{2}$ Biehl, Montgomery, Neher, Pickering, and Roesch, Rev. Mod. Phys. 20, 360 (1948).

${ }^{3} \mathrm{~S}$. E. Forbush and Isabelle Lange, Researchers of the Department of Terrestrial Magnetism, Vol. XIV,
}

for all latitudes, yet the time intervals involved are short since the B-29 travels over 300 m.p.h. at this altitude. Past experience has shown that except on rare occasions fluctuations over a period of a few hours are negligible. Further, the experimental values obtained on the return flight indicated no detectable change from the values going south. As an additional check, Dr. S. E. Forbush of the Carnegie Institution of Washington kindly furnished us with data taken with his continuously recording meters at Huancayo, Peru, and Cheltenham, Maryland. These showed no appreciable disturbances during this period.

In order to avoid changing longitude it was planned to fly along longitude $80^{\circ}$ West which conveniently passes near Florida, Panama, and Peru, all of which have landing fields for B-29's. The 80th meridian further has the advantage of nearly coinciding with a geomagnetic longitude.

\section{EXPERIMENTS PERFORMED}

Although measurement of the latitude effect of the radiation coming in near the vertical was the primary object of this flight, a number of other measurements also were made. Since the flight took place along a nearly constant geomagnetic longitude, the heavy cosmic-ray telescopes could be rotated about a single, north-south horizontal axis to measure first the radiation coming in from the west, and then the radiation coming in from the east. Since the heading of the plane was always nearly north or south, these counter telescopes would be pointing toward geomagnetic east or west at all times.

A list of experiments performed both on the trip to Peru and return as well as on a high flight over Peru is given below. The range of latitude was from $64^{\circ}$ geomagnetic North to the geomagnetic equator along longitude $80^{\circ}$ geographic West. 


\section{A. Pressure Altitude 30,000 Ft. $\left(310 \mathrm{G} \mathrm{Cm}^{-2}=3.10 \mathrm{M}\right.$ of Water)}

1. Latitude effect-Continuous measurements both going and coming--

a. Vertical radiation: Two independent telescopes; no shielding.

b. Vertical radiation: One telescope; $10 \mathrm{~cm}$ of lead absorber.

c. Vertical radiation: One telescope; $20 \mathrm{~cm}$ of lead absorber.

d. East-West effect at $45^{\circ}$ : Two independent telescopes; no absorber.

e. East-West effect at $45^{\circ}$ : One telescope; $10 \mathrm{~cm}$ of lead absorber.

f. East-West effect at $45^{\circ}$ : One telescope; $20 \mathrm{~cm}$ of lead absorber.

g. Wide showers: Four trays of counters. Maximum extension 3.5 meters.

h. Total radiation: Continuously recording ionization chamber. Same as used in our sea level and airplane surveys.

2. Other Experiments at 30,000 fl.-

a. East-West effect at equator: Measurements made with no lead, 10 and $20 \mathrm{~cm}$ absorber at zenith angles of $0^{\circ}, 22 \frac{1}{2}^{\circ}, 45^{\circ}, 67 \frac{1}{2}^{\circ}, 90^{\circ}$ in the East-West plane.

b. Zenith angle measurements: Made in NorthSouth plane at $48^{\circ}$ geomagnetic North on flight from Provo, Utah, to Springfield, Illinois, along a constant geomagnetic latitude. No absorber, 10 and $20 \mathrm{~cm}$ of lead absorber. Zenith angles: $0^{\circ}, 22 \frac{1}{2}^{\circ}, 45^{\circ}, 67 \frac{1}{2}^{\circ}, 90^{\circ}$. These same measurements were repeated on the EastWest flight along the geomagnetic equator from longitude $80^{\circ}$ West to Lima, Peru.

c. Side showers: To provide data on the corrections due to showers from the sides to be made to the counting rate of the counter telescopes the center tray of counters on two telescopes was displaced sideways outside the telescope aperture. This was done both at northern latitudes and at the equator.

\section{B. Pressure Altitude 36,000 Ft. (235 G $\mathrm{Cm}^{-2}$ ) over Peru}

1. Azimuthal experiments-Two hexagonal courses flown, one hexagon rotated $30^{\circ}$ with respect to the other, thus giving data every $30^{\circ}$ in azimuth. Measurements made with no lead, $10 \mathrm{~cm}$ and $20 \mathrm{~cm}$ of lead absorber. Zenith angles: $0^{\circ}, 22 \frac{1}{2}^{\circ}, 45^{\circ}, 67 \frac{1}{2}^{\circ}$.

2. Wide showers also measured.

\section{Miscellaneous Experiments}

1. Counting rate versus air pressure-At nearly all times when the plane was ascending, all telescopes were turned vertically and records were kept of outside air pressure versus time.

2. Calibration checks-On several occasions on the flight down and return all telescopes were turned vertically and long runs made for intercomparison.

\section{DESCRIPTION OF APPARATUS}

The apparatus used in these experiments consisted essentially of three parts; the cosmic-ray telescopes, the scaling circuits, and the recording equipment.

\section{A. The Cosmic-Ray Telescopes}

These were the same, with slight modifications, as those previously used by us in balloon experiments. ${ }^{2}$ They consisted of three nearly equally spaced trays in triple coincidence each containing eight Geiger-Müller tubes. Each tray had an area of $650 \mathrm{~cm}^{2}$, the spacing between extreme trays being about $80 \mathrm{~cm}$. This spacing was reduced from the previously used value of one meter in order (1) to fit into the frame four equally spaced trays and (2) to increase the counting rate. This arrangement permitted the top three trays to be used as one telescope and the lower three as another. The center two trays thus were common to each telescope. This arrangement was used in the telescopes measuring the East-West effect and in the vertical telescopes containing 10 and $20 \mathrm{~cm}$ of lead. The two used for the total vertical intensity were separate and mounted in individual frames.

The Geiger tubes, described elsewhere, ${ }^{4}$ were made of copper-plated steel. The steel was $0.025 \mathrm{~cm}$ thick bent to form a tube $3.3 \mathrm{~cm}$ in diameter and $25 \mathrm{~cm}$ long. The five walls necessary for a particle to penetrate to register as a triple coincidence thus corresponded to an average thickness of $0.20 \mathrm{~cm}$ of iron or very closely $1.6 \mathrm{~g} \mathrm{~cm}^{-2}$.

Figure 1 shows schematically the arrangement used in the plane. The two frameworks holding the two sets of 3 trays each, forming the two telescopes without lead absorber, were mounted in the rear corners of the pressurized section of the B-29. The other three frame-

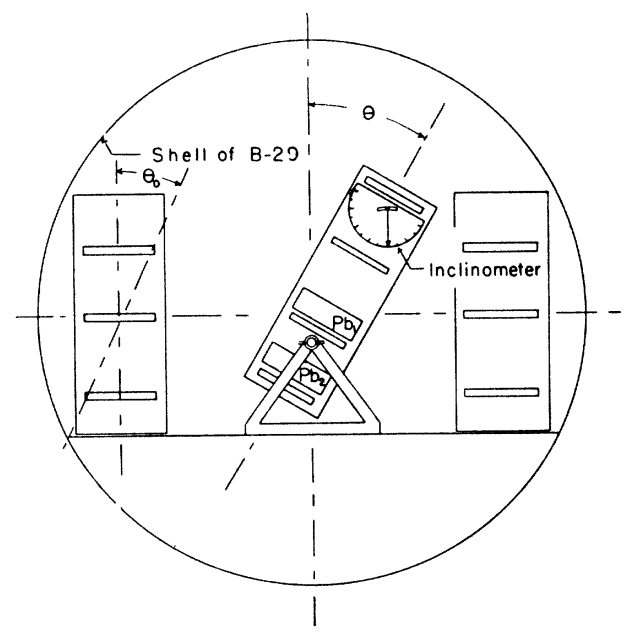

FIG. 1 Disposition of cosmic-ray telescopes in rear pressurized section of the B-29.

${ }^{4}$ Biehl, Montgomery, Neher, Pickering, and Roesch, Rev. Mod. Phys. 20, 354 (1948). 
works were mounted along the center of the plane. Two of these latter were used for East-West measurements. In each of these cases, $\mathrm{Pb}_{1}$ was missing while $\mathrm{Pb}_{2}$ was $10.2 \mathrm{~cm}$ of lead in one and $20.3 \mathrm{~cm}$ of lead in the other. For measuring the absorption of the vertical radiation, $\theta$ in Fig. 1 was kept at $0^{\circ}$ while $\mathrm{Pb}_{1}$ was $10.2 \mathrm{~cm}$ of lead and $\mathrm{Pb}_{2}$ was another $10.2 \mathrm{~cm}$ of lead.

In the case of the East-West arrangement, for example, the top three trays would respond to the total radiation incident within a certain solid angle while the lower three trays would count only those particles that had sufficient energy to penetrate either 10 or $20 \mathrm{~cm}$ of lead incident within the same solid angle.

The trays were nearly square, being $23.1 \mathrm{~cm}$ by $28.5 \mathrm{~cm}$. Allowing for cracks between counters, the effective area was about $600 \mathrm{~cm}^{2}$. With the spacing used, the extreme half-angle $\theta_{0}$ in one plane was $16^{\circ}$ and in the other $20^{\circ}$. In the telescopes that were tilted the counters were mounted so that the $16^{\circ}$ angle was in the vertical plane.

An inclinometer with a pointer was mounted on the sides of the two frames that were rotated. The graduated circles on these read to $\frac{1}{4}^{\circ}$ and the sensitivity of the bubble was such that a rotation of $\frac{1}{4}^{\circ}$ could easily be detected.

Each telescope frame was covered with 0.5 -mm sheet aluminum. This performed a threefold purpose: (1) electrical shielding, (2) mechanical rigidity, and (3) physical protection. The amplifiers and coincidence circuits were the same as used on balloon flights previously. ${ }^{2}$

The two frames holding the four East-West telescopes and the single frame holding the two vertical telescopes containing lead were mounted along the center of the rear pressurized section of the B-29. The width of the plane was just sufficient to allow the East-West sets to be tipped until they were horizontal. The total weight of each of the two heavier pieces with the $20 \mathrm{~cm}$ of lead was approximately $200 \mathrm{~kg}$.

\section{B. The Scaling Circuits}

The maximum coincident counting rates expected were approximately 1000 per minute. With the mechanical registers used a scale of 4 to 1 was sufficient to reduce the randomness and average rate of counting to a value where a negligible number of counts would be missed.

Each scaling circuit consisted of a multivibrator pulse shaper (Fig. 2), two scale of two circuits and a final multivibrator for driving the register. Germanium crystal diodes were used between stages of the scaling circuit for isolation purposes. The frequency response of the complete circuit was measured to be approximately 50 kilocycles per second without the mechanical register attached. The register itself would follow 50 evenly spaced pulses per second.

Ten scaling units were built, nine of which were used at any one time. A switch on the input side permitted a pulse of variable frequency to be fed in, to check the scaling circuits and registers. Another switch permitted the scaling circuit to be eliminated and the single pulses would then be recorded on the register, thus permitting checks to be made on the scaling factor.

To avoid effects of moisture in the tropics, all circuits were dipped in hot paraffin.

\section{The Recording Unit}

The dials of the nine registers, a clock, and pertinent information written on a small card were all photographed once every minute on a $35-\mathrm{mm}$ film.

\section{Auxiliary Equipment}

1. A wide shower experiment was performed using four trays in quadruple coincidence. The physical arrangement is shown in Fig. 3. Four trays were necessary to reduce the accidental rate to an inappreciable value.

2. The pressure altitude of the plane was obtained by

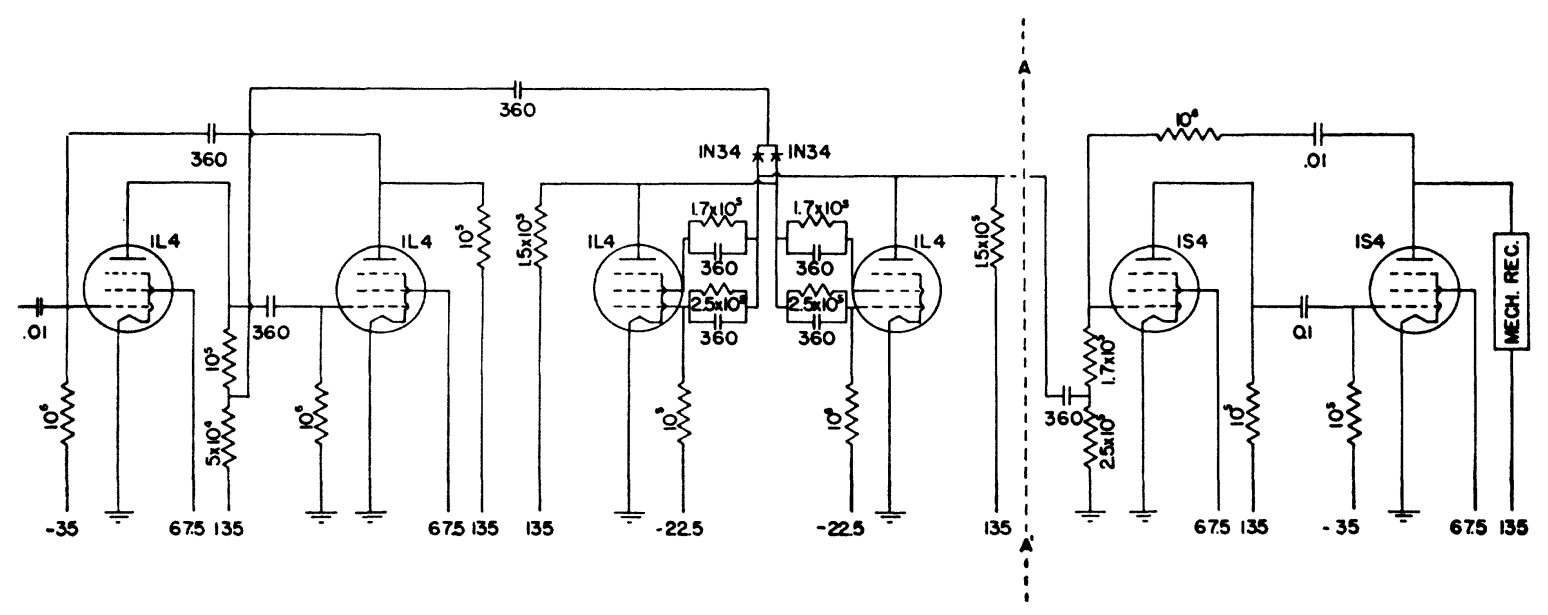

FIG. 2. Input and output multivibrators for the scales-of-two. At $A A^{\prime}$ another scale-of-two was used. 
two methods: (a) The plane's altimeter and (b) a Friez aircraft continuously recording barograph suspended in the bomb bay. The plane's altimeter was calibrated prior to the flight by the Air Forces in terms of the standard atmosphere. ${ }^{5}$

3. An ionization chamber like that used in previous years on airplanes and on ships at sea gave a continuous record of the total intensity. ${ }^{6}$ This instrument was placed in the pressurized section of the plane along with the other equipment and as far removed from radioactive dials as possible.

The temperature of the rear pressurized section of the B-29 was kept at about $20^{\circ} \mathrm{C}$ during the flights and the air pressure was maintained at a value corresponding to about 10,000-ft. elevation.

\section{INTERNAL CALIBRATIONS}

All telescope data were adjusted by suitable correction factors to read what set No. 1 (one of the vertical, unshielded telescopes) would have read in the same circumstances. These calibration factors were determined as follows:

On several occasions, both on the ground and in the air, the four unshielded sets were measuring the same radiation while the cosmic-ray intensity was constant for a sufficient time to yield an accurate ratio between counting rates. There is no significant difference between the two methods of comparing the rates. Averaging the ratios so obtained using the reciprocals of the probable errors as weighting factors provided the calibration factors.

For the calibration of the telescopes containing lead absorber, data taken in the plane on the ground were used. These data gave the counting rate with the lead removed but the iron boxes which held the lead still in place. An experiment in which iron sheets were inserted between the trays of the unshielded telescopes gave the necessary correction for the metal boxes, so that the above counting rates could be compared with those of set No. 1 (see Fig. 4).

This last procedure failed in one case due presumably to the failure of a counter in the set. However, on other occasions its counting rate could be compared with another set with the same absorber.

The results of the internal calibrations were as follows :

Set No
3
4
5
6
7
8
9

Ratio of No. 1 to this set
$0.979 \pm 0.004$
$0.981 \pm 0.007$
$1.037 \pm 0.009$
$1.000 \pm 0.007$
$1.037 \pm 0.006$
$1.011 \pm 0.006$
$1.019 \pm 0.016$

(Set No. 2 was used to measure extensive showers.)

\footnotetext{
${ }^{5}$ Report No. 538 of the National Advisory Committee for Aeronautics.

' Robert A. Millikan and H. Victor Neher, Phys. Rev. 50, 15 (1936).
}

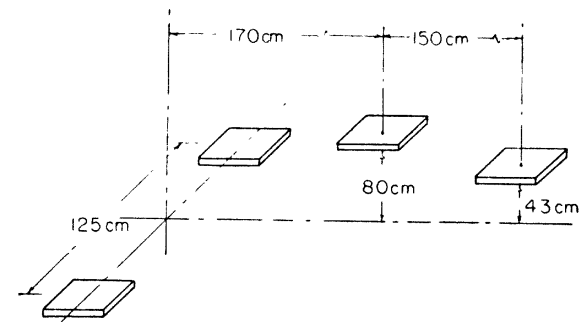

FIG. 3. Four trays of 8 counters each with $600 \mathrm{~cm}^{2}$ area used to measure extensive showers had a maximum extension of about $3.5 \mathrm{~m}$.

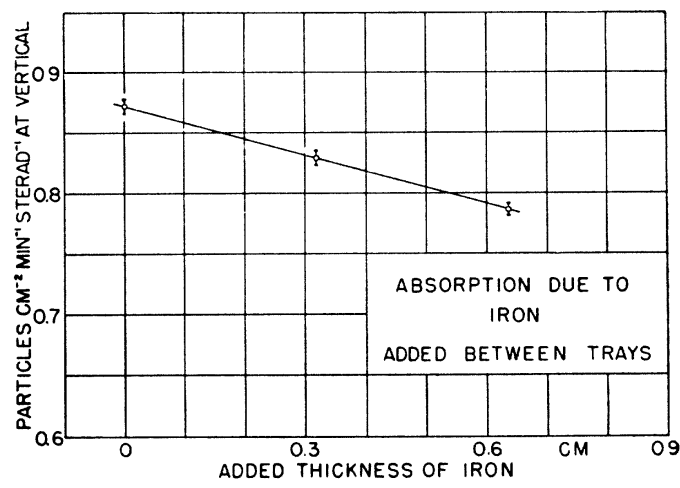

FIG. 4. Adding iron sheets between the trays at Inyokern gives an absorption of $2.0 \pm 0.2$ percent $\mathrm{g}^{-1} \mathrm{~cm}^{2}$.

The indicated probable errors are those computed from statistical errors in the calibration. The above comparison gives a good experimental check of the similarity of the geometries of the various telescopes.

\section{CORRECTIONS}

The following is a list of corrections to the different arrangements that need to be considered:
A. Accidental counting rates.
B. Deadtime of counters.
C. Effect of side showers.
D. Variation in pressure (altitude).
E. Efficiency of recording circuit.
F. Variation in zenith angle of the East-West sets.
G. Effects of finite telescope aperture.
H. Effect of finite thickness of counter walls and absorption in walls of plane.

These corrections will be discussed individually.

(A) The accidental counting rate was important only at the high altitudes and is due almost entirely to the real double coincidence rate between any two trays and the single counting rate of the third tray.* Using the known geometry of the counters and the counting rate of a single counter at the equator ${ }^{7}$ we can compute this accidental rate at different latitudes. The computation depends slightly on the amount of lead between the

* This particular correction was not applied to the results using this same equipment reported in Rev. Mod. Phys. 20, 366 (1948). We are grateful to G. Cocconi for calling our attention to this omission.

${ }^{7}$ H. V. Neher and W. H. Pickering, Phys. Rev. 61, 407 (1942). 
TABLE I. Total correction in counts per minute due to showers for telescopes with no lead for zenith angle $z .^{*}$

\begin{tabular}{cccccc}
\hline \hline Latitude & $z=0$ & $22 \frac{1}{2}^{\circ}$ & $45^{\circ}$ & $67 \frac{1}{2}^{\circ}$ & $90^{\circ}$ \\
\hline $50^{\circ} \mathrm{N}$ & $100 \pm 10$ & $92 \pm 10$ & $85 \pm 15$ & $80 \pm 15$ & $76 \pm 10$ \\
0 & $66 \pm 7$ & $61 \pm 7$ & $57 \pm 10$ & $53 \pm 10$ & $50 \pm 7$ \\
\hline
\end{tabular}

* No correction made for local side showers for telescopes with lead absorber.

TABLE II. Corrections to be applied to zenith angle data for finite opening of telescope (no lead absorber).

\begin{tabular}{ccccc}
\hline \hline $\begin{array}{c}\text { Zenith } \\
\text { angle }\end{array}$ & $\begin{array}{c}48^{\circ} \text { North } \\
(3.1 \text { m of water })\end{array}$ & N-S plane & $\begin{array}{c}0^{\circ}(2.3 \mathrm{~m} \text { of water }) \\
\text { West }\end{array}$ & East \\
\hline 0 & $0 \%$ & $0 \%$ & $0 \%$ & $0 \%$ \\
$22 \frac{1}{2}^{\circ}$ & +1.0 & +0.1 & +0.3 & +0.2 \\
$45^{\circ}$ & -2.0 & +1.1 & +1.1 & +0.6 \\
$67 \frac{1}{2}^{\circ}$ & -23 & -8.0 & -8.1 & -9.5 \\
\hline
\end{tabular}

trays and on the zenith angle distribution at the high altitudes but this we know from measurements made during these flights.

If $I$ represents the number of ions $\mathrm{cm}^{-3} \mathrm{sec}^{-1}$ in air at N.T.P. as measured with our thin-walled balloon ionization chambers, ${ }^{8}$ then we compute the relative number of accidentals for any one of the unshielded telescopes to be

$$
R_{a}=1.52 \times 10^{-4} I .
$$

This gives a value of -1.5 percent as a correction for the telescopes with no lead at the northern latitudes and -1.0 percent at the equator, both at $3.10 \mathrm{~m}$ of water equivalent.

(B) The correction for deadtime was determined experimentally. ${ }^{4}$ Again this correction depends on the counting rates of the individual counters which will be proportional to $I$, the ionization measured by a thinwalled ionization chamber. The relative correction is given by

$$
R_{a}=4.3 \times 10^{-4} I \text {. }
$$

This gives a correction of +4.0 percent at the northern latitudes at $30,000 \mathrm{ft}$. and +2.6 percent at the equator.

(C) The correction for side showers is somewhat more difficult to make. These showers will probably be due mostly to small, local events originating in the material in the immediate neighborhood such as the equipment and the structure of the plane or in the air outside.

When the East-West telescopes were turned over so that their axes made $90^{\circ}$ with the vertical, the counting rate, except for scattering, should, because of the finite aperture of the telescope, be due to particles that have penetrated a minimum of $11 \mathrm{~m}$ of water equivalent (1100 $\mathrm{g} \mathrm{cm}^{-2}$ of air). The radiation in this direction should be of about the same composition as that found at sea level, namely 30 percent soft and 70 percent penetrating particles. The 10 or $20 \mathrm{~cm}$ of lead in the telescopes should stop the non-penetrating particles

Bowen, Millikan, and Neher, Phys. Rev. 53, 855 (1938). and further, because of the shielding, it would be expected that the correction due to local side showers for the telescopes containing lead would be negligible. The average of the counting rates at northern latitudes with 10 and $20 \mathrm{~cm}$ of lead when the telescopes were horizontal was 21 per minute. We estimate from our extended shower measurements that about 7 counts per minute of these will be due to extended showers. Since in this position the telescopes receive particles from both ends, the number of penetrating particles incident from one end from $16^{\circ}$ above the horizon to the horizon is 7 per minute. This is to be compared with about 800 per minute at the vertical for the telescopes with no lead or an average of 180 per minute at the vertical for 10 and $20 \mathrm{~cm}$ of lead. This rate when the telescopes with lead were horizontal is not far from the counting rate to be expected from the underwater data on counting rates due to radiation near the vertical coming through an equal amount of matter.

Taking this evidence that the effect of local side showers on the telescopes containing lead is negligible and assuming that the radiation coming in at these large angles is similar to that found near the vertical at sea level and below, we can compute what the rate should be in the unshielded telescopes when horizontal. This turns out to be 26 per minute. The actual counting rate under these conditions was 102 per minute. The difference, or 76 per minute, we will assume is due to local side showers.

To find the effect of side showers at other zenith angles we can use the data on the counting rate with the center tray displaced outside the aperture of the telescope. At the northern latitudes at 30,000 ft. the counting rate with the center tray displaced on the telescope with no lead was 44 per minute when the telescope was vertical. When tipped over at $45^{\circ}$ this

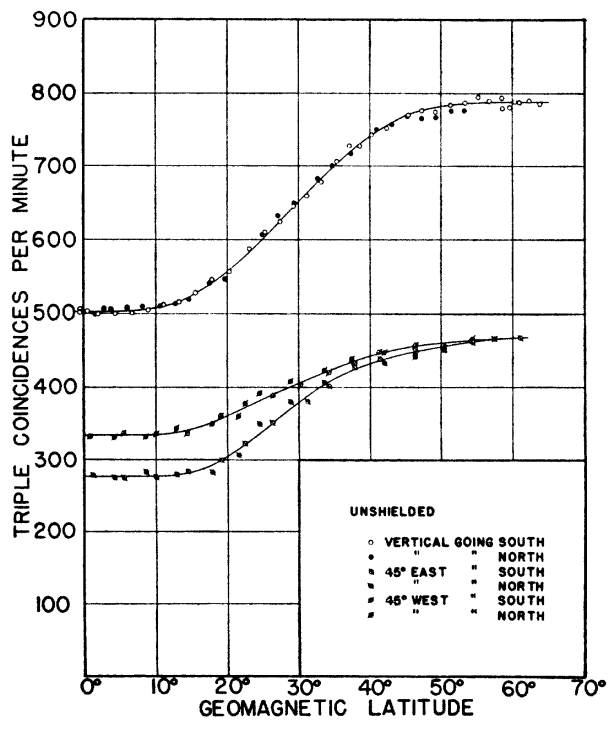

FIG. 5. Latitude effect in the radiation at the vertical, $45^{\circ} \mathrm{West}$ and $45^{\circ}$ East with no absorber. No corrections applied. 
TABLE III.

\begin{tabular}{rll}
\hline $\begin{array}{c}\text { Flight } \\
\text { No. }\end{array}$ & \multicolumn{1}{c}{ Route } & \multicolumn{1}{c}{ Time } \\
\hline 4 & Inyokern-Chicago & May 26, 1948, 0334-1004* \\
5 & Chicago-54 N-Tampa & May 29, 1948, 1146-2100 \\
6 & Tampa-Panama & June 2, 1948, 1522-2334 \\
7 & Panama,-Lima, Peru & June $4,1948,0945-1839$ \\
8 & Above Peru & June $6,1948,1505-2130$ \\
9 & Lima-Panama & June 8, 1948, 1537-2324 \\
10 & Panama-Tampa & June 9, 1948, 1738-0130 \\
11 & Tampa-Chicago & June 10, 1948, 2044-0328 \\
12 & Above Illinois and Wisconsin & June 12, 1948, 1758-0118 \\
13 & Scott Field-Inyokern & July 1, 1948, 1405-2240
\end{tabular}

* Greenwich civil time.

rate dropped to 29 per minute. At the equator this latter went down to 20 per minute. (The fact that the latitude effect in these showers is about the same as for the total intensity means that most of them are not of the extended variety which show no latitude effect (see Fig. 10).)

R. A. Montgomery of this laboratory ${ }^{8 a}$ has analyzed the side showers at sea level and concludes that most of the events with three trays of counters are due to two time-associated particles, one passing through one tray and one passing through the other two considered as a wide angle telescope. Assuming a zenith angle distribution the same for these small showers as for the total radiation, it is possible to make an estimate, for a known geometry, of the dependence of such events on zenith angle and on the position of the center tray. Superimposed on the effect due to these small side showers will be the extensive or wide showers whose frequency of occurrence we also measured during these flights. The contribution of these to our telescopes we will take to be 7 per minute, independent of spacing, zenith angle, or latitude. The above kind of analysis leads us to the following scheme for the total correction due to showers for the telescopes without lead at different zenith angles (Table I). Corrections are in counts per minute and are to be subtracted from the original data (see Fig. 5). Estimates of the errors involved are based on the uncertainties in applying shower data to our particular geometry. We assume that these showers will, to a first approximation, show no asymmetry at the equator.

It should be pointed out that the relative importance of side showers should be considerably less with the large area trays made up of small diameter Geiger tubes here used, than with the usual single Geiger tube telescopes of small spacing. This is due, (1) to the relatively smaller projected areas at large angles to the axis of the telescope and (2) to the relatively large spacing between trays. It is readily seen that a telescope of large size will be less subject to showers from material in the immediate neighborhood since in general the shower is concentrated in a small solid angle.

\footnotetext{
8a R. A. Montgomery, Phys. Rev. 75, 1407 (1949).
}

(D) Variation in pressure due to variations in the height of the plane were negligible. From previous data we calculate that at $30,000 \mathrm{ft}$. at northern latitudes the change in cosmic-ray intensity for a change of $100 \mathrm{ft}$. in altitude is about 0.6 percent for the total intensity at the vertical. The altimeter indicated that the plane was seldom off more than $100 \mathrm{ft}$. and then not for any appreciable time. Since most of our data are averaged over a period of 30 minutes such fluctuations should be negligible.

(E) A simple calculation shows that the efficiency of

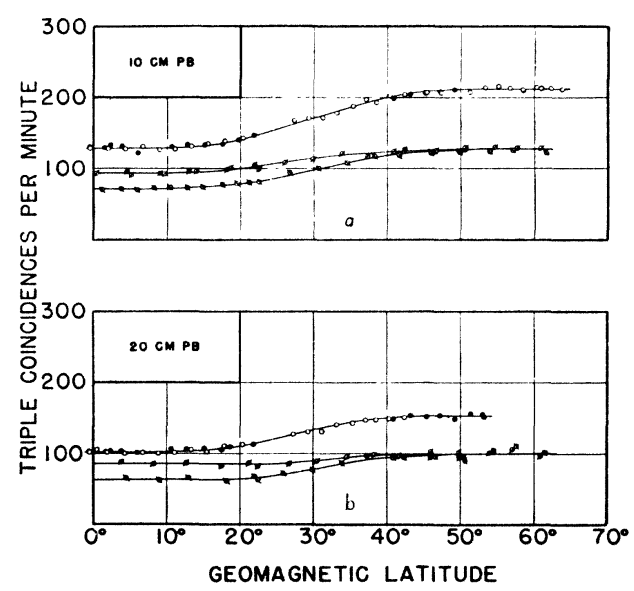

FIG. 6. Latitude effect in the radiation at the vertical, $45^{\circ}$ West and $45^{\circ}$ East for that part which can penetrate 10 and $20 \mathrm{~cm}$ of lead. No corrections applied.

the recording circuit with the scale of 4 and the register used was nearly 100 percent for the highest counting rates encountered.

(F) Effect of variation of zenith angle on the EastWest sets may be calculated roughly as follows: The zenith angle dependence at $48^{\circ}$ North and 30,000 ft. with no absorber may be represented by

$$
k=k_{0} \cos ^{1.7} z \text {, }
$$

where $z$ is the zenith angle and $k_{0}$ is the counting rate at the vertical. At $z=45^{\circ}$ a variation in $z$ of $\pm 1^{\circ}$ will result in a variation in counting rate of 3 percent.

Except on rare occasions the roll of the plane was not more than $\pm 0.5^{\circ}$ and the statistical fluctuation in the roll of the plane is estimated at $\pm 0.25^{\circ}$. Thus this correction may be ignored.

The effect of variation of zenith angle on the vertically directed telescopes was at all times completely negligible.

The geometrical alignment of the trays in the eastwest telescopes as well as the positioning of the inclinometer were checked by tipping the telescopes $45^{\circ}$ North then $45^{\circ}$ South on the ground at Inyokern and comparing the two counting rates. As originally aligned, no difference between these two rates could be found within the probable errors.

(G) Effect of finite opening of telescopes: The tele- 


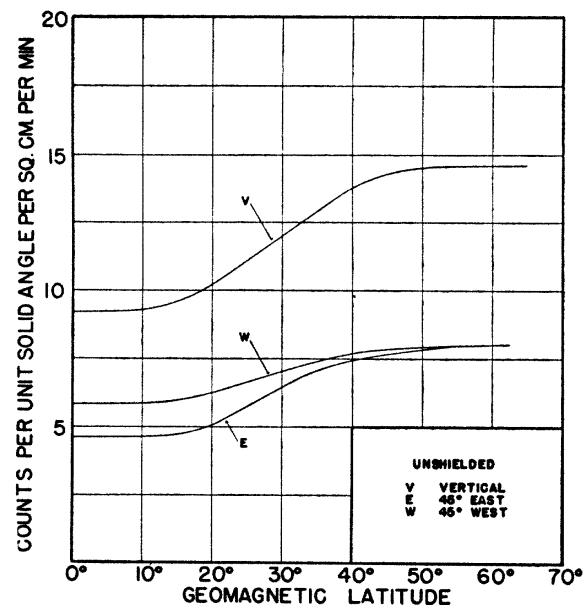

FIG. 7. Data of Fig. 5 reduced to absolute values.

scopes here used, when tipped at an angle of $z$ to the zenith, will respond to particles coming in within the angles $z \pm 16^{\circ}$ in the vertical plane and $\pm 20^{\circ}$ in azimuth. The mass of air thus varies over the aperture, depending on the direction. If it is assumed that the variation of the intensity over the aperture can be represented by the vertical intensity curve for the same mass of air then a double graphical integration will give the correction for the aperture here used.

This graphical integration has been performed at $48^{\circ}$ North and at the equator. Representative values are shown in Table II. The correction for the case where the telescope is pointed in the vertical direction has been taken into account in the absolute calibration.

It should be pointed out that for telescopes with apertures much larger than are here used the corrections for large zenith angles become quite important. A finite aperture in either azimuth or the vertical plane increases the counting rate at large zenith angles compared with that at the vertical at altitudes below the maximum of the counts $v s$. depth curve.

(H) The five wall-thicknesses of the counters that a particle needed to penetrate to trip a triple coincidence set totaled up to an average over the area of $1.6 \mathrm{~g} \mathrm{~cm}^{-2}$ of iron. The material of the plane added about another $1 \mathrm{~g} \mathrm{~cm}^{-2}$. To find the effect of adding iron between the trays of counters on the ground two additional thicknesses were used. The results are shown in Fig. 4. The relative slope of this line is $2.0 \pm 0.2$ percent $\mathrm{g}^{-1}$ $\mathrm{cm}^{2}$ of iron.

(I) Summary of Corrections.-A list of corrections made to the original data is as follows:
A. Accidentals
B. Deadtime of counters
C. Side showers
No lead absorber
With lead absorber
D. Finite aperture (all zenith angles except $\left.67 \frac{1}{2}^{\circ}\right)$

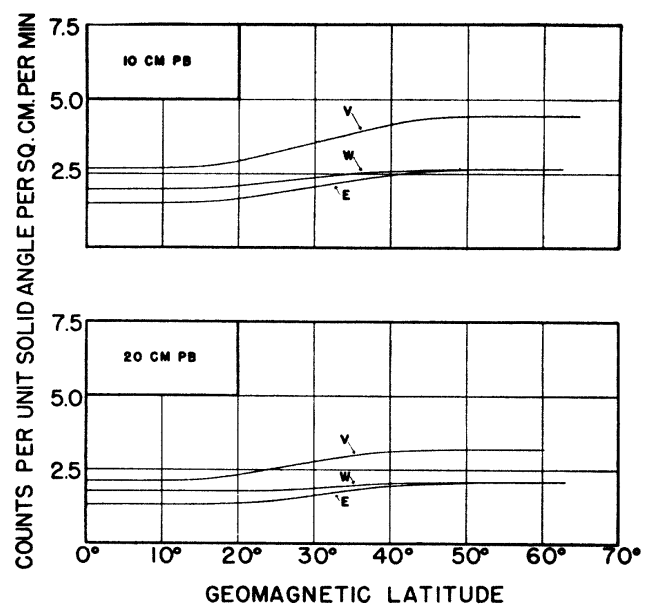

FIG. 8. Data of Fig. 6 reduced to absolute values.

\section{REDUCTION TO ABSOLUTE VALUES}

After the data for each telescope were corrected for accidentals, deadtime, side showers, and finite aperture, the resultant counting rate was multiplied by an appropriate factor to reduce the rate to an absolute value. Montgomery of this laboratory has made a careful determination of the absolute intensity of cosmic rays at sea level and in Pasadena with the counters here used and finds a value of 0.697 ionizing particles $\mathrm{cm}^{-2}$ min. $^{-1}$ steradian $^{-1}$ at the vertical at sea level. This is in close agreement with the value given by Greisen. ${ }^{9}$

Comparing our geometry with that used by Montgomery we arrive at the result that our counting rates must be multiplied by 0.0208 to reduce them to particles $\mathrm{cm}^{-2}$ min. $^{-1}$ steradian $^{-1}$. If we, on the other hand, assume that the intensity of cosmic rays at sea level has not changed during the 3 or 4 months between the determinations made by Montgomery and the time

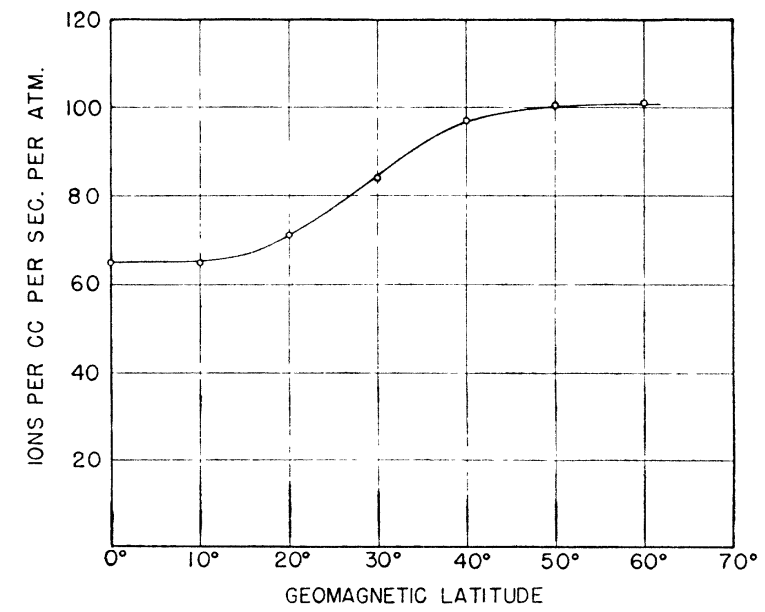

FIG. 9. Latitude effect of the total radiation as measured with a continuously recording ionization chamber.

${ }^{9}$ K. I. Greisen, Phys. Rev. 63, 323 (1943). 
just prior to these flights we can find the multiplying factor necessary merely by placing our unshielded telescopes outdoors in Pasadena. By such a method we find a multiplying factor of 0.0205 . As a mean of these two we take the value of 0.0206 .

\section{EXPERIMENTAL PROCEDURES}

\section{A. Route of the B-29}

The airplane left Inyokern at 7:34 p.m. local time on May 26, 1948, and reached 30,000 ft. in one hour. The flight plan called for flying straight to Provo, Utah, follow a geomagnetic latitude from there to Springfield, Illinois, and then turn north to Chicago. On May 29 we flew northeast from Chicago to latitude $54^{\circ}$ North, longitude $80^{\circ}$ West, which placed us over the lower end of Hudson Bay. Turning south and flying at a constant pressure altitude of $30,000 \mathrm{ft}$. we followed the $80^{\circ} \mathrm{W}$ meridian to Charleston, South Carolina, where we dropped to lower elevation because of a storm, landing at Tampa, Florida. June 2 we took off from Tampa, retraced our route to Charleston to connect on to the 30,000 -ft. point on the previous flight, then turning south we landed at Panama. The beginning of each flight was in this way always connected on the end of the preceding flight to leave no gaps in the records. A summary of the flights is given in Table III.

\section{B. Procedures during Flight}

(1) All telescopes were pointed vertically while gaining altitude and while descending for all flights. The rate of rise was about the same as for the balloon flights we had made in the past. This gave additional data at several latitudes on the counting rate versus pressure with and without lead absorber.

(2) The dials of the 9 registers, besides being photographed every minute, were read visually every 10 minutes and the rates of counting computed immediately to give a constant check on the behavior of the equipment.

(3) Frequent checks were made on the apparatus

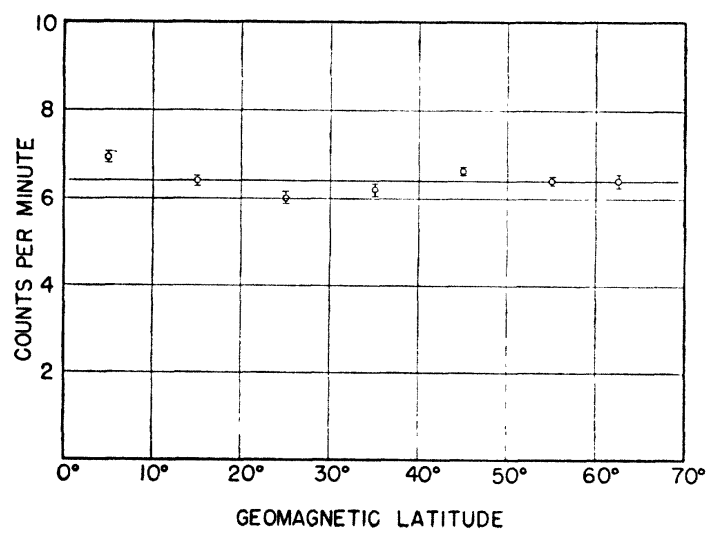

FIG. 10. Extensive showers show no latitude effect at $3.10 \mathrm{~m}$ of water equivalent air pressure.

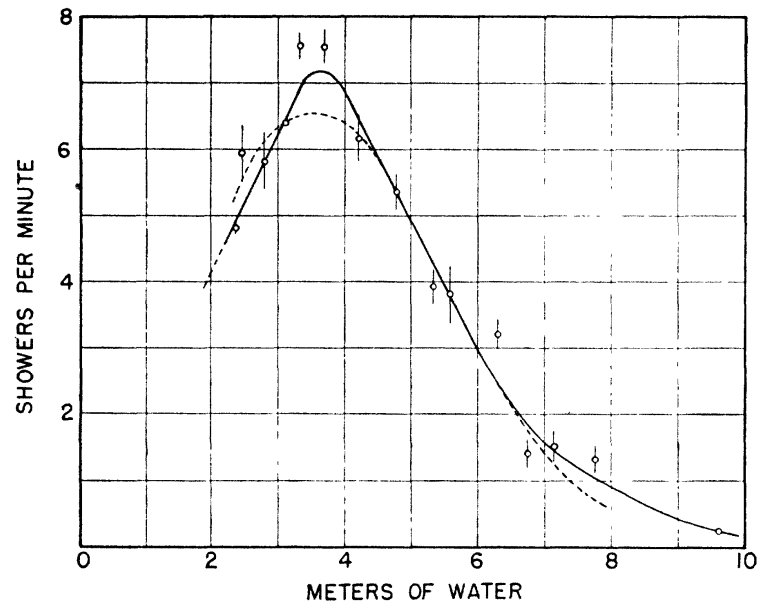

FIG. 11. Extensive showers here measured show absorption of $150 \mathrm{~g} \mathrm{~cm}^{-2}$ beyond the peak. Dashed curve is that obtained by Kraybill (Phys. Rev. 73, 632 (1948)).

not only while in the air but on the ground. An oscilloscope was used to determine the behavior of both Geiger tubes and circuits. With the 187 miniature radio tubes and 152 Geiger tubes some difficulties were to be expected. Actually four Geiger tubes failed during the entire trip. Interruptions caused by these failures were in all cases never serious.

(4) During the flights north and south, the two observers (H. V. Neher and A. T. Biehl) shifted the two frameworks holding the four East-West telescopes every ten minutes from $45^{\circ}$ East (or West) to $45^{\circ}$ West (or East). Approximately 3 seconds of time were necessary to make this shift.

(5) During the azimuthal flight over Peru at $2.35 \mathrm{~m}$ of water equivalent the East-West sets were tipped to a given angle with the vertical and left there for two full hexagonal courses of $360^{\circ}$. Two full hexagonal courses were again flown with the two sets tipped at other angles. The two successive hexagons were rotated $30^{\circ}$ with respect to each other to permit readings every $30^{\circ}$ in azimuth. The time of flight along each side of a hexagon was approximately $10 \mathrm{~min}$.

(6) A constant check was maintained on the reading of the altimeter and the heading of the plane. An inter-phone system permitted communication at any time with the pilots as well as other members of the crew.

(7) The position of the plane was determined by an experienced navigator.

(8) While flying over water the actual height of the plane was frequently determined with the radio altimeter.

\section{EXPERIMENTAL RESULTS}

\section{A. Latitude Effect of the Vertical Radiation}

(1) The counting rates of the two vertical telescopes without absorber, as a function of geomagnetic latitude, are given in the upper curve of Fig. 5. Each point on 


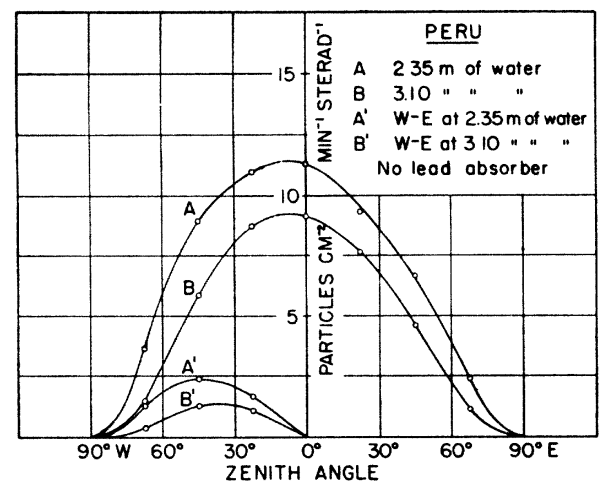

FIc. 12. Zenith angle dependence in the East-West plane at two different elevations over Peru with no lead absorber.

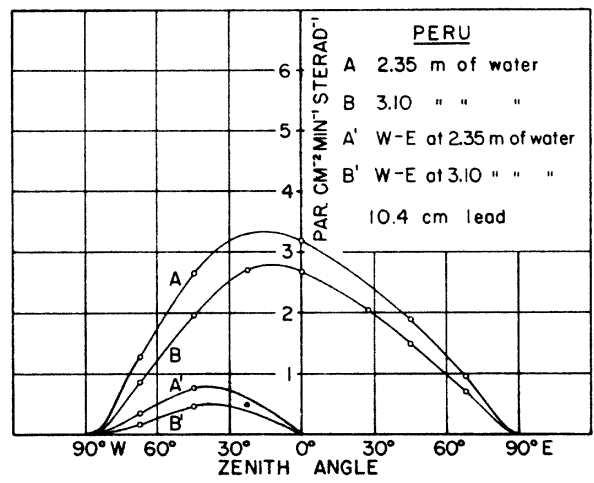

Fig. 13. Zenith angle dependence in the East-West plane at two different elevations over Peru with $10 \mathrm{~cm}$ lead absorber.

the curve represents the average of the two sets each averaged for a 30 minute interval of time. At the northern latitudes each point is determined by nearly 50,000 counts giving a statistical probable error of about \pm 0.3 percent. Thus the error in each point from the number of counts is given approximately by the radii of the circles (or dots). No corrections have been applied to these data.

The open circles represent the results of the trip south and the solid circles for the trip north. It is seen that in general the agreement is quite good indicating that the absolute value of the radiation did not change appreciably in the intervening 4 to 11 days (see Table III). There is, however, an indication of some fluctuations on the return flight at about $50^{\circ}$ North as seen in Fig. 5.

(2) The latitude effects taken with the two telescopes with 10 and $20 \mathrm{~cm}$ of lead between the second and third trays of counters are given in the top curves of Fig. 6(a) and (b). The open and solid points again represent the values going south and north respectively. Since only one telescope was used for each curve, the statistical probable error over the 30 -minute period at the northern latitude was \pm 1 percent or about one-half the radius of a circle (or dot).

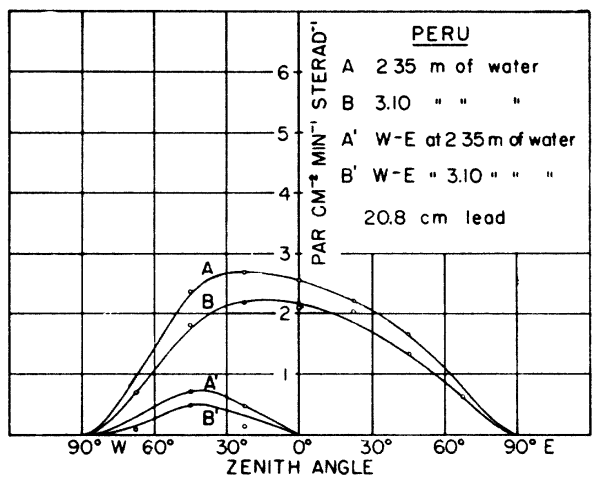

FIG. 14. Zenith angle dependence in the East-West plane at two different elevations over Peru with $20 \mathrm{~cm}$ of lead absorber.

\section{B. Latitude Effect of the East-West Effect}

In Figs. 5 and 6 the lower pairs of curves give the results obtained with the counter telescopes pointed east and west, without and with lead, respectively. Again the average for each point was over three intervals of 10 minutes each. The data without lead were the averages of two independent telescopes, each alternately pointing $45^{\circ}$ East half the time and $45^{\circ}$ West the other half. Thus each point without lead represents the counting rate obtained from about 28,000 counts at the northern latitudes.

The data with 10 and $20 \mathrm{~cm}$ of lead were obtained with a single telescope for each. The statistical probable error in each point is thus about \pm 1.2 percent or again about one-half the radius of one of the circles (or dots).

It should be noted that in certain regions of the curves with lead as shown in Fig. 6 some of the data are missing. These gaps are due to failure of the equipment but fortunately do not appear to affect the general behavior of the curves.

The above results on the latitude effect without and with lead are now corrected for side showers and multiplied by the factor 0.0206 to reduce the data to particles $\mathrm{cm}^{-2}$ min. $^{-1}$ steradian $^{-1}$. The reduced curves are given in Figs. 7 and 8. The side shower correction applied to the telescopes without lead amounted to, at northern latitudes, a reduction of 12.8 percent for the readings of the vertical telescopes and 18.5 percent for those tipped at $45^{\circ}$ to the vertical.

TABLE IV. East-West asymmetry over Peru, zenith angle $45^{\circ}$.

\begin{tabular}{ccc}
\hline $\begin{array}{c}\text { M of water } \\
\text { equivalent }\end{array}$ & $\begin{array}{c}\text { Thickness of } \\
\text { absorber }\end{array}$ & $\begin{array}{c}\text { West excess* } \\
(\%)\end{array}$ \\
\hline 3.10 & $0 \mathrm{~cm}$ & $23.3 \pm 1.1$ \\
3.10 & $10 \mathrm{~cm}$ & $27.6 \pm 1.3$ \\
3.10 & $20 \mathrm{~cm}$ & $30.4 \pm 1.5$ \\
2.35 & $0 \mathrm{~cm}$ & $29.1 \pm 1.4$ \\
2.35 & $10 \mathrm{~cm}$ & $33.0 \pm 1.6$ \\
2.35 & $20 \mathrm{~cm}$ & $35.4 \pm 1.7$ \\
\hline
\end{tabular}

* Computed from the difference between West and East divided by the average. 


\section{Latitude Effect of the Total Radiation}

The results obtained from the ionization chamber, reduced to ions $\mathrm{cm}^{-3} \mathrm{sec}^{-1}$ at one atmosphere of air are given in Fig. 9. The error in each point is approximately \pm 1.5 percent as determined from the scatter of the readings of the five-minute individual discharges on the film.

\section{Latitude Effect of Extensive Showers}

The four trays of eight counters each, connected to give quadruple coincidences, gave a counting rate of about 6 per minute. The points given in Fig. 10 are averaged over $10^{\circ}$ of latitude. The statistical probable errors are indicated by the vertical lines through the points.

\section{E. Altitude Effect of Extensive Showers}

The altitude dependence of extended showers is shown by the solid line in Fig. 11. Since there appears to be no latitude dependence at high altitudes for wide showers, the results shown have been averaged from data collected while the plane was gaining altitude from several locations. The point at $3.10 \mathrm{~m}$ of water is the average for the whole trip while the one at $2.35 \mathrm{~m}$ of water is the result of about 2000 counts. The geometry of the four trays of counters used is shown in Fig. 3.

\section{F. East-West Effect at the Equator}

Preliminary results on the East-West effect at the equator have been reported previously ${ }^{10}$ but for completeness are included here. The data without lead at the two elevations over Peru are given in Fig. 12. Corrections for accidentals, deadtime of the Geiger

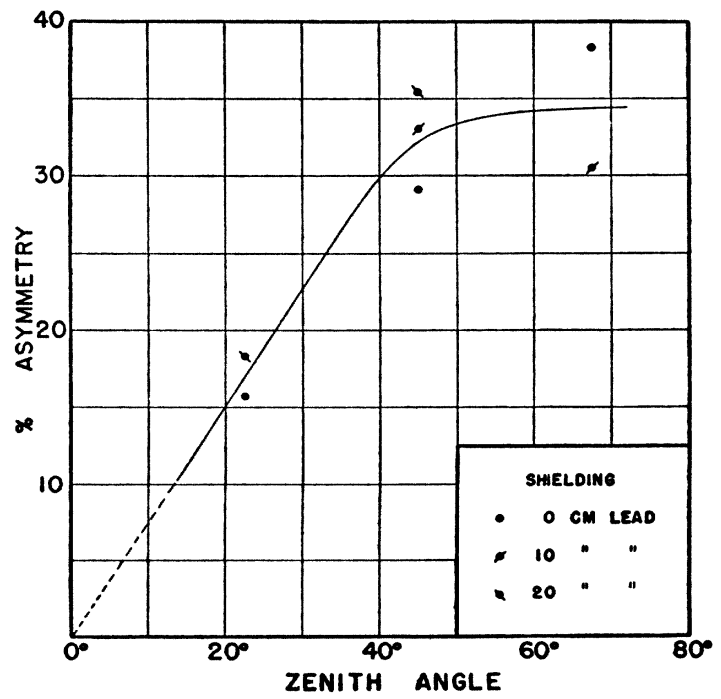

FIG. 15. Percentage West excess as a function of zenith angle with and without lead absorber.

${ }^{10}$ Biehl, Neher, and Roesch, Phys. Rev. 75, 688 (1949). tubes, side showers, and finite opening of the telescope have been made.

The results with 10 and $20 \mathrm{~cm}$ of lead are given in Figs. 13 and 14, respectively, for the two different elevations over Peru. The total correction for accidentals and deadtime was about 1 percent while no corrections were made for side showers.

The data at $22 \frac{1}{2}^{\circ}$ with $10 \mathrm{~cm}$ of lead and at $67 \frac{1}{2}^{\circ}$ with $20 \mathrm{~cm}$ of lead are missing at the higher elevation because of lack of time.

The percentage west excess at $45^{\circ}$ is given in tabular form in Table IV.

For other zenith angles the percentage West excess is plotted against angle for the higher elevation over Peru in Fig. 15.

\section{G. Azimuthal Experiments at the Geomagnetic Equator over Peru}

The variations in azimuth of the radiation at different zenith angles, $z$, without and with lead absorber are

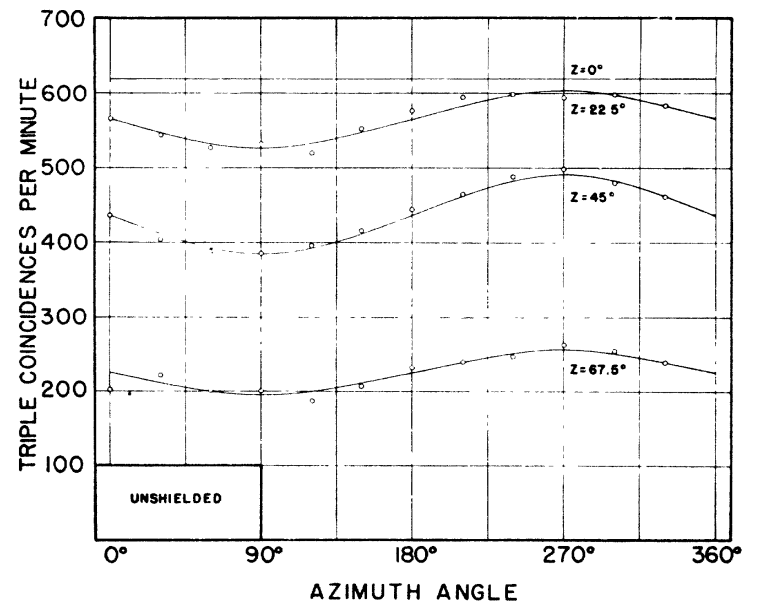

FIG. 16. Azimuthal effect at $2.35 \mathrm{~m}$ of water over Peru with no lead absorber. No corrections applied.

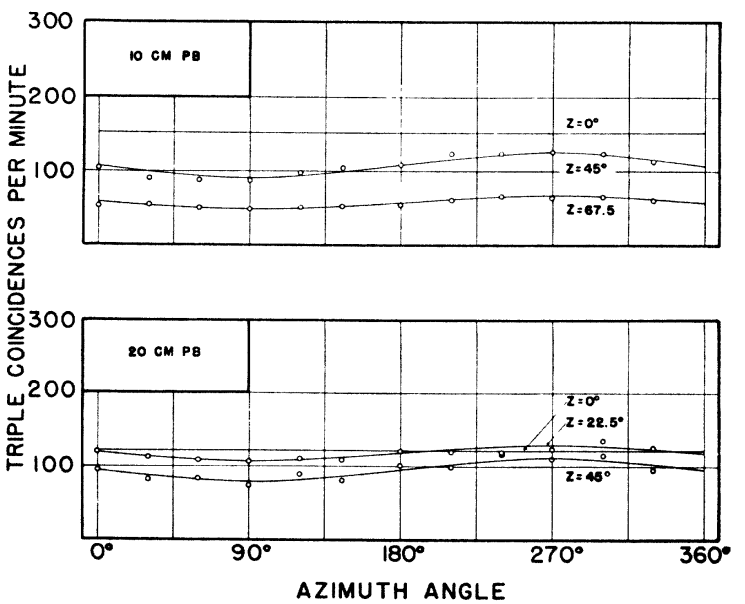

Fig. 17. Azimuthal effect at $2.35 \mathrm{~m}$ of water over Peru with 10 and $20 \mathrm{~cm}$ of lead absorber. No corrections applied. 
given in Figs. 16 and 17, respectively. Each point given resulted from averaging the counting rate over a period of about 10 minutes. This results, for example, for no lead absorber in a statistical error of \pm 0.7 percent for each point at zenith angle of $45^{\circ}$. In the case of the

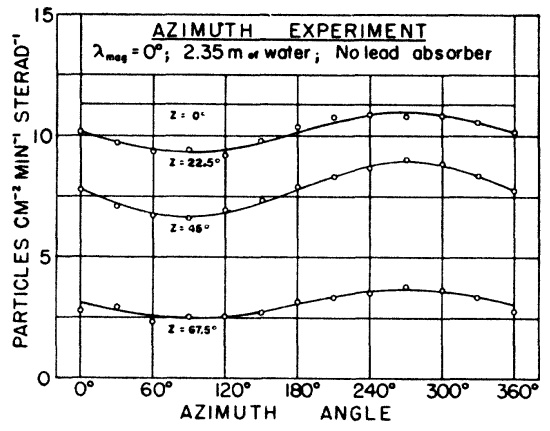

Fig. 18. Data of Fig. 16 reduced to absolute values.

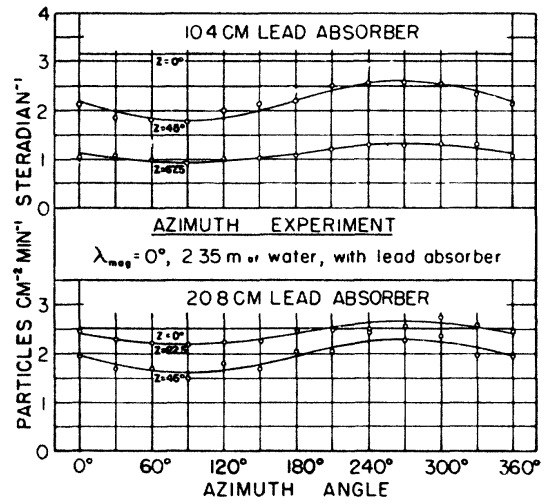

Fig. 19. Data of Fig. 17 reduced to absolute values.

telescopes with lead the probable error of each point at $45^{\circ}$ is about \pm 2.1 percent.

When corrections are made for side showers and finite opening of the telescopes as previously described and the counting rates reduced to absolute values the results obtained are given in Figs. 18 and 19.

\section{H. Zenith Angle Measurements}

At the geomagnetic latitude of $48^{\circ}$ North while flying at 30,000 ft. between Provo, Utah, and Springfield, Illinois, the telescopes were tilted north and south to find the dependence of counting rate on zenith angle. These results are plotted in Fig. 20 for no lead absorber, $10 \mathrm{~cm}$ and $20 \mathrm{~cm}$ of lead. Corrections for side showers, accidentals, deadtime, and finite opening of the telescope have been made to these data.

\section{Intensity versus Air Pressure}

The results shown in the logarithmic plots of Figs. 21 and 22 were obtained as follows: The upper curve with no lead was obtained on gaining altitude on two occasions when leaving Chicago. The data at the equator with no lead were obtained from two flights while gaining altitude out of Lima. The data for the $10 \mathrm{~cm}$ and $20 \mathrm{~cm}$ of lead absorber were obtained in each case from the flights leaving Chicago and Lima.

The results with lead are in fair relative agreement with the values of Gill, Schein, and Yngve ${ }^{11}$ and of Swann and Morris. ${ }^{12}$

\section{J. Altitude versus Air Pressure}

The B-29 was equipped with a radio altimeter with which true height could be readily determined while

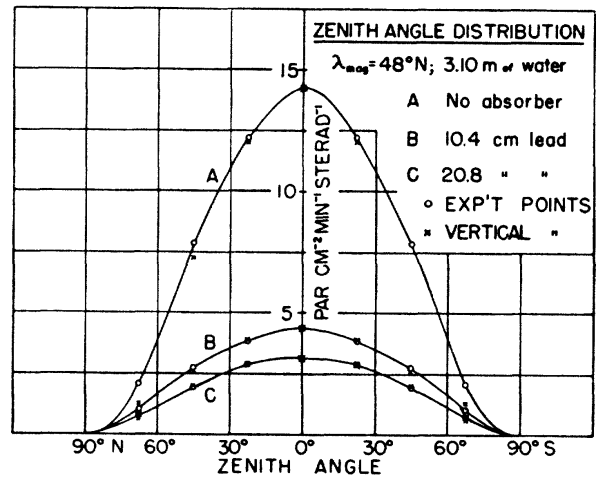

Fig. 20. Zenith angle dependence in North-South plane at $48^{\circ}$ geomagnetic north at a pressure of $3.10 \mathrm{~m}$ of water. Value at $67 \frac{1}{2}^{\circ}$ with no lead through same mass of air as at vertical is about 50 percent higher.

flying over water. The differences between the true height and the pressure height, as obtained from the pressure altimeter which was calibrated in terms of the standard atmosphere, are plotted at several different

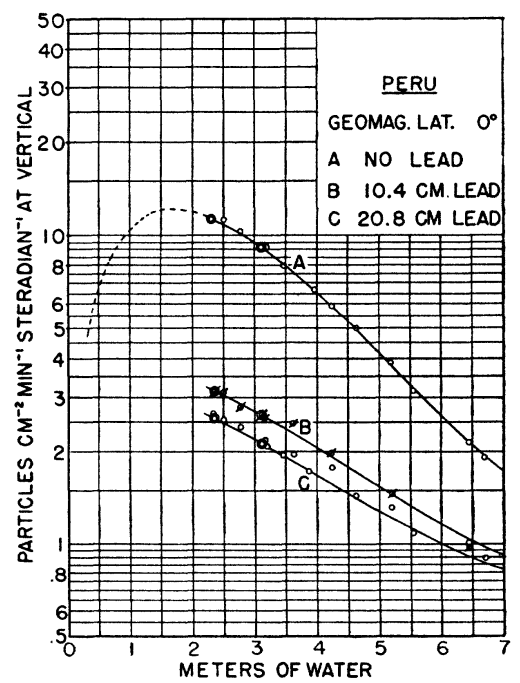

FIg. 21. Intensity at the vertical over Peru. Extended curve with no lead is that obtained from balloon data.

${ }^{11}$ Gill, Schein, and Yngve, Phys. Rev. 72, 733 (1947).

12 Second Annual Report of Bartol Research Foundation; ONR Contract N6ori-144, September 15, 1947. 
latitudes in Fig. 23. This curve shows a maximum somewhat north of the geographic equator. This is not surprising since the sun was nearly at its northernmost excursion when these flights were made.

\section{DISCUSSION}

\section{A. Latitude Effect of the Radiation at the Vertical}

If the latitude effect is defined as the ratio of the change of the radiation in going from the far northern latitudes to the equator at constant barometric pressure, to that existing at the far northern latitudes, then the values given in Table $\mathrm{V}$ are obtained.

The conclusion drawn from these values is that the latitude effect for the total radiation at the vertical is very closely the same as for the radiation that can penetrate either 10 or $20 \mathrm{~cm}$ of lead. This is the same conclusion arrived at by Millikan and Neher ${ }^{13}$ for the radiation at sea level in their world survey with ionization chambers. On the several occasions in this survey when unshielded instruments and those shielded with $10 \mathrm{~cm}$ of lead were compared, the same latitude effect was obtained with each. This means that not only at sea level but also at a pressure of $\frac{1}{3}$ atmosphere the latitude effect in the penetrating and non-penetrating components is the same. The same conclusion for the radiation at sea level has been arrived at by Morris, Swann, and Taylor $^{14}$ for their sea level data with counters.

At sea level where the penetrating and non-penetrating components are in equilibrium an equal latitude effect for the two is easily understandable. Since, as discussed by Rossi, ${ }^{15}$ it seems that the soft component cannot arise at high altitudes entirely from decay of

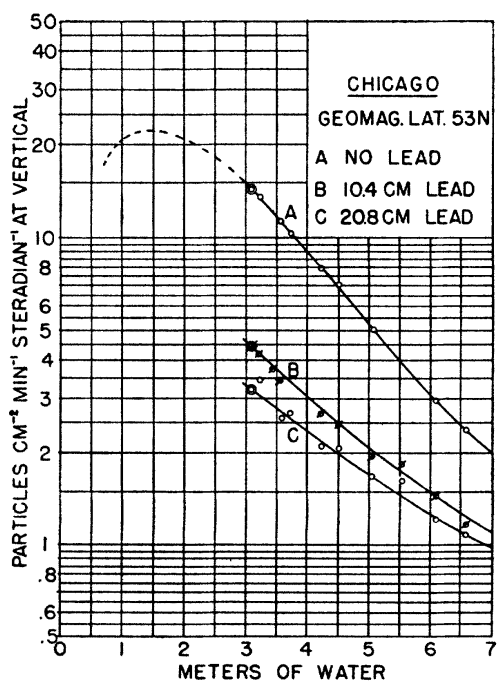

FIG. 22. Intensity at the vertical at $53^{\circ}$ geomagnetic North. Extended curve with no lead is obtained from balloon data taken at Omaha.

${ }^{13}$ R. A. Millikan and H. V. Neher, Phys. Rev. 50, 15 (1936)

${ }_{14}$ Morris, Swann, and Taylor, Phys. Rev. 72, 1263 (1947).

${ }^{15}$ B. Rossi, Rev. Mod. Phys. 20, 573 (1948).

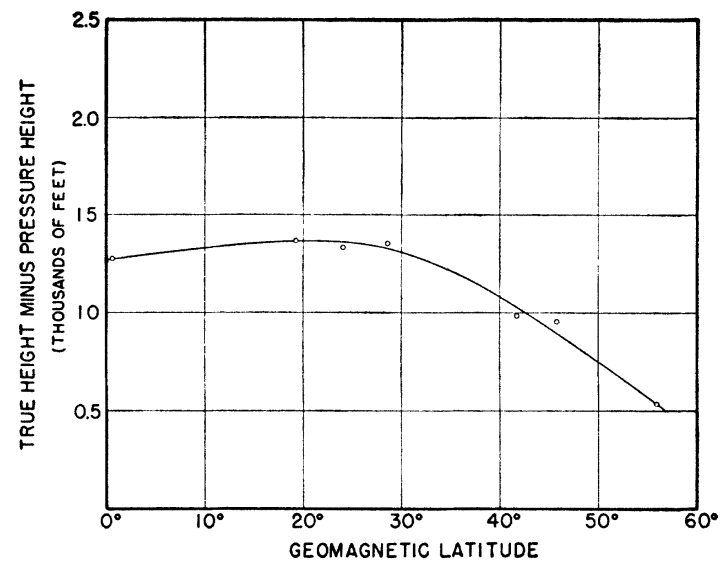

Fig. 23. At the time this flight was made (June 1948) the true altitude was always greater than the pressure altitude.

mesons or collision processes there appears to be some other intimate relationship between the genesis of penetrating and non-penetrating particles which is independent of the energy of the initiating primary particle.

Another way of stating the above experimental fact is to say that there is the same ratio for a given pressure altitude between penetrating and non-penetrating particles at far northern latitudes as at the equator. For approximately the same mass of intervening air, covering a range of average momentum of the incoming particles of a factor of 4 , some selected values are given in Table VI. This shows very clearly that in spite of the much different energy of the incoming particles that there are the same relative numbers of penetrating to non-penetrating particles.

This constant ratio of penetrating to non-penetrating particles, for the same mass of air traversed, which is independent of the energy of the primary particle up to a momentum of at least $23 \mathrm{Bev} / \mathrm{c}$ and which is also found for the radiation remaining beyond $23 \mathrm{Bev} / \mathrm{c}$, argues for a similar type of incident particle which is responsible for the background radiation found at the equator. The effects of decay are here ignored. Later it will be shown that such effects are small at zenith angles of $45^{\circ}$.

\section{B. Adjustment of Former Altitude Curves}

As stated in the introduction, the original purpose of this flight was to fix one point on all the counter telescope curves taken with balloons by the California Institute group thus giving the result of having made all the balloon flights simultaneously.

Of the curves available those made at Saskatoon (Canada), Omaha, Fort Worth, ${ }^{16}$ Acapulco $^{17}$ (Mexico) and Bangalore ${ }^{18}$ (India) were chosen as being the most

${ }^{16}$ For data at Saskatoon, Omaha, and Fort Worth, see Rev. Mod. Phys. 20, 360 (1948).

${ }^{17}$ Millikan, Neher, and Pickering, Phys. Rev. 63, 234 (1943).

${ }^{18}$ H. V. Neher and W. H. Pickering, Phys. Rev. 61, 407 (1942). 
TABLE V.

\begin{tabular}{cc}
\hline Absorber & $\begin{array}{c}\text { Latitude effect at vertical at } \\
\text { 3.10 m of water equivalent }\end{array}$ \\
\hline 0 & 0.37 \\
$10.4 \mathrm{~cm} \mathrm{~Pb}^{*}$ & 0.40 \\
$20.8 \mathrm{~cm} \mathrm{~Pb}$ & 0.34 \\
\hline
\end{tabular}

* Actual single thickness of lead was $10.2 \mathrm{~cm}$ (4 inches). Additional amount given corresponds to mass equivalent of iron holder for lead.

TABLE VI.

\begin{tabular}{ccccccc}
\hline \hline & & & \multicolumn{3}{c}{$\begin{array}{c}\text { Penetrating } \\
\div \text { total }\end{array}$} \\
$\begin{array}{c}\text { Geomag. } \\
\text { lat. }\end{array}$ & $\begin{array}{c}\text { Zenith } \\
\text { angle }\end{array}$ & Mass of air & $\begin{array}{c}\text { (Bev/c) } \\
\text { min. }\end{array}$ & $\begin{array}{c}\text { (Bev/c) } \\
\text { ave.* }\end{array}$ & $\begin{array}{c}10 \mathrm{~cm} \\
\mathrm{~Pb}\end{array}$ & $\begin{array}{c}20 \mathrm{~cm} \\
\mathrm{~Pb}\end{array}$ \\
\hline $38^{\circ} \mathrm{N}$ & $0^{\circ}$ & $3.1 \mathrm{~m}$ of water & 5.4 & 17 & 0.30 & 0.24 \\
$0^{\circ}$ & $0^{\circ}$ & $3.1 \mathrm{~m}$ of water & 13.2 & 40 & 0.29 & 0.23 \\
$0^{\circ}$ & $45^{\circ} \mathrm{E}$ & 3.3 m of water & 23.0 & 70 & 0.28 & 0.24
\end{tabular}

* Computed from a differential numbers distribution $\mathrm{p}^{-2.5} \mathrm{dp}$.

consistent and reliable. The normalization was accomplished by multiplying all values at a given latitude by a factor which made the point at $3.10 \mathrm{~m}$ of water equivalent agree with the present B-29 data. This procedure is justified by the following: (1) If the fluctuations at high altitudes are due to fluctuations of cosmic rays as a whole, the procedure is certainly justified. (2) If these fluctuations are due to a change in the numbers of incident particles near the minimum allowable momentum at a given latitude the procedure is justified by the fact that curves taken at two latitudes not too far apart are closely similar. A more detailed adjustment could be carried through in principle but is not found necessary due to (a) the amount of adjustment in most cases is small and (b) the errors in the experimental data do not justify further refinement. Since the areas under the curves are of primary interest, ${ }^{19}$ this adjustment involves the least error when made near the peaks of the curves.

In transforming the Bangalore curve to the geomagnetic equator in Peru considerable adjustment needs to be made because of the longitude effect. However, as will be seen in Fig. 21, two very well determined points in Peru help to fix the curve near the maximum. Also it is found that when plotted logarithmically all these curves are close to straight lines between 3 and $7 \mathrm{~m}$ of water but whose slopes depend on the latitude; i.e., upon the momenta of the incident particles. This information allows an interpolation for intermediate latitudes and altitudes.

The curves of Fig. 24 represent then, the best estimate that can be made at the present time of the curves that would have been obtained at these various locations had all the flights been made simultaneously. The agreement of the present values with those obtained in $1947^{16}$ may be seen from the fact that the adjusted value at $3.10 \mathrm{~m}$ of water is now 14.55 particles $\mathrm{cm}^{-2}$

${ }^{19}$ H. V. Neher and W. H. Pickering, Phys. Rev. 61, 410 (1942). min..$^{-1}$ steradian $^{-1}$ at Saskatoon compared with 14.37 then obtained.

A derived set of curves may be obtained from the above by plotting the counting rate as a function of latitude for constant air pressure. This has been done in Fig. 25. These results indicate that while the latitude effect appears to cease at about $53^{\circ}$ at $30,000 \mathrm{ft}$. in going north, there is still some latitude effect at the higher altitudes beyond this latitude.

The conclusions we draw from these data are as follows: (1) There is no sharp cut-off of the primary radiation at least up to $60^{\circ}$ geomagnetic North. While the energy being brought in by the primary particles whose momentum is less than $3 \mathrm{Bev} / \mathrm{c}$ is relatively small, there is no indication that the differential numbers distribution of the primary particles passes through a maximum. (2) That within the limits of these latitude experiments the momentum distribution of the primary particles has no sharp breaks between approximately 2 and $13 \mathrm{Bev} / \mathrm{c}$.

The conclusion that there is no sharp cut-off of particles of low momentum is further strengthened by the balloon data taken with ionization chambers by Bowen, Millikan and Neher in $1937 .^{20}$ The flights made at Saskatoon, Canada, and Omaha, Nebraska, covered a period of two weeks during the latter part of August and the first part of September of that year. The maximum deviation from the mean at the maximum of the curve of any one 4-minute reading of the three flights made at Saskatoon on August 14, 16, and 17 was 1.8 percent. The similar measure of the consistency of the 5 flights made at Omaha on August 21 through September 5 was a maximum deviation of 2.7 percent for any one point. The measured difference in the two curves at the maximum was 7 percent. Further, the data of the Carnegie Institution meters at Huancayo and Cheltenham show that this period was very quiet in the lower part of the atmosphere.

This lack of cut-off at very high altitudes and latitudes is further confirmed by the recent work of Frier, Ney, and Oppenheimer ${ }^{21}$ at altitudes of 70,000 to $90,000 \mathrm{ft}$. using photographic emulsions. They find evidence for particles having energies at the top of the atmosphere as low as $0.5 \mathrm{Bev}$ per nucleon at a latitude of $55^{\circ}$ North.

\section{Zenith Angle Effect at the Equator}

Lemaitre and Vallarta ${ }^{22}$ have given the boundaries to the cones for certain limiting momenta of the primary particles at the equator. Of particular interest here is the cone whose boundary passes through the zenith. This boundary is tangent to the North-South direction at the zenith and bends toward the West at the larger zenith angles. From an application of Liouville's theorem it is concluded that at all zenith angles along

${ }^{20}$ Bowen, Millikan, and Neher, Phys. Rev. 53, 855 (1938).

21 Freier, Ney, and Oppenheimer, Phys. Rev. 75, 991 (1949).

${ }^{22}$ G. Lemaitre and M. Vallarta, Phys. Rev. 50, 500 (1936). 
this boundary line the same numbers of particles per unit time of the same momentum distribution are incident per unit area per unit solid angle at the top of the atmosphere.

If, then, from the curves of Figs. 18 and 19 values are picked off that correspond to these azimuth angles of the same limiting momentum, the results shown in Fig. 26 are obtained. These curves correspond to those taken at $48^{\circ}$ North as shown in Fig. 20 and give the zenith angle effect at $2.35 \mathrm{~m}$ of water at the geomagnetic equator.

It is possible to get the zenith angle effect also at $3.10 \mathrm{~m}$ of water at the equator if one assumes a sinusoidal variation in azimuth such as is found at $2.35 \mathrm{~m}$ of water. Experimental data were taken at the azimuth angles $0^{\circ}, 90^{\circ}, 180^{\circ}$, and $270^{\circ}$ at the lower elevation and the assumption of a sinusoidal variation in azimuth involves only a slight correction to the North-South values. The results for no lead absorber are given in Fig. 27 together with the similar data at the same pressure taken at $48^{\circ}$ North. The values of $48^{\circ}$ North may be fitted rather closely with the empirical relation

$$
I / I_{0}=\cos ^{1.70} z
$$

while at the equator the approximate relation is

$$
I / I_{0}=\cos ^{1.62} z \text {. }
$$

Plotted in Figs. 20 and 27 are the intensities at the vertical as measured with the same telescopes under the same mass of air. It will be noticed that the agreement is quite good where lead absorber is used but there is some discrepancy with no lead at $45^{\circ}$ and still more at $67 \frac{1}{2}^{\circ}$, the vertical values being in all cases lower, for the same mass of intervening air, than those measured at an angle to the zenith.

There are at least two phenomena that must be taken into account when comparing counting rates under the

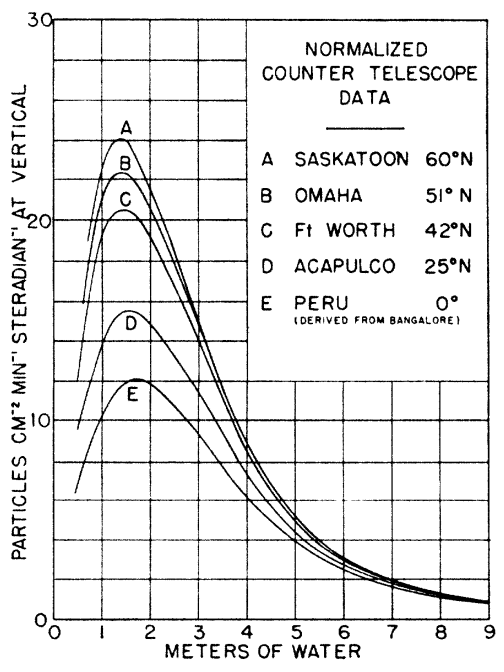

FIG. 24. Former balloon counter telescope flights normalized to present airplane data at $3.10 \mathrm{~m}$ of water. same mass of air but where the path lengths are different. These are decay of the meson and scattering. The effect of decay may be either to add to or subtract from the total number of ionizing particles through the same mass of air but over a greater path. If the unstable particle decays while still having considerable kinetic energy remaining, the decay electron will form a shower and thus may increase the numbers of particles that would otherwise exist. On the other hand the effect of decay should always tend to lower the corresponding numbers of penetrating particles.

Without further experimental data or an extended theoretical analysis, it is not possible at the present time to estimate the effects of decay or of scattering. Perhaps fortuitously, the combination of these two effects at these altitudes, results in nearly perfect agreement at all zenith angles for the case of the penetrating component as is seen from Fig. 20.

In connection with the effect of decay on the intensity of the meson component and how the extension of the atmosphere might affect such decay as a function of latitude, Fig. 23 is included. It will be noticed that at the altitude flown $(30,000 \mathrm{ft}$.) the maximum excursion of the curve giving the difference between the true height and the pressure height is only $830 \mathrm{ft}$. Most of this change takes place at the northern latitudes.

\section{Effect of Atmospheric Scattering}

The effect of scattering in the atmosphere is to decrease the intensity of the secondary particles in directions where the intensity is high and to increase the intensity in the directions where it is low. Thus the zenith angle distribution found near sea level would, by this principle, be more peaked at the vertical with less intensity at large zenith angles were scattering not present.

Some indication as to the importance of scattering

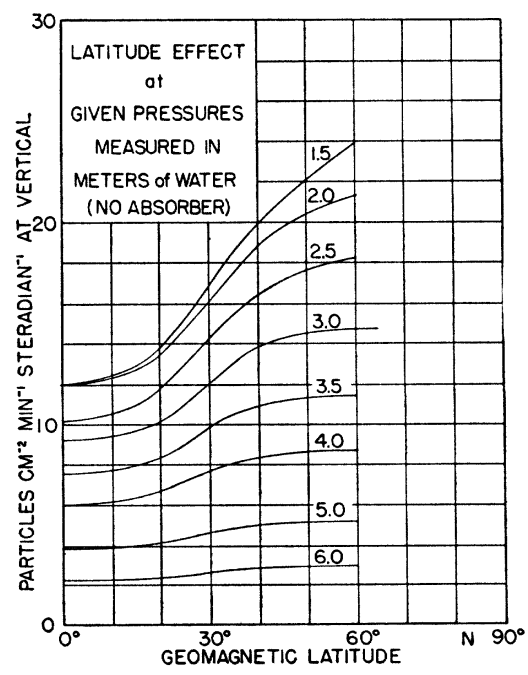

FIG. 25. Derived curves from Fig. 24 showing latitude effect at given depths in the atmosphere. 


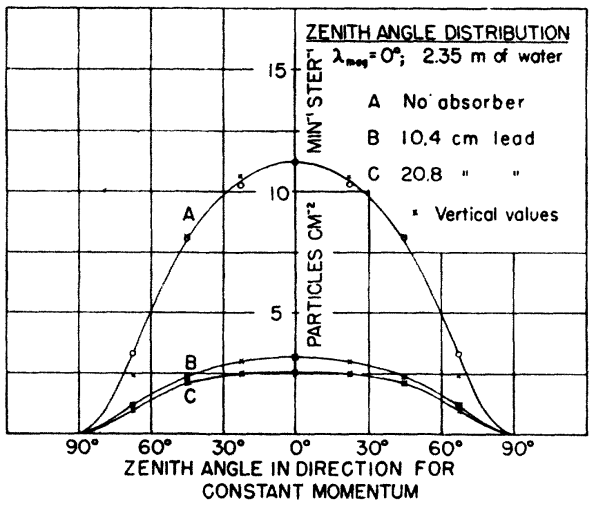

FIg. 26. Zenith angle dependence at $2.35 \mathrm{~m}$ of water in the direction of constant incident momentum at the equator.

may be obtained by comparing the intensity measured at large zenith angles at high altitudes with that measured near the vertical at lower altitudes and under the same mass of air. Winckler, Stroud, and Schenck ${ }^{23}$ for example find twice the intensity at $67 \frac{1}{2}^{\circ}$ to the vertical with no lead absorber as at the vertical for one-half atmosphere of air in each case. Although no estimate, from their geometry, of the effect of finite opening of their telescope can be made, it seems reasonable that some of the excess intensity measured at this large zenith angle is due to scattering.

From our own data we see from Figs. 20 and 27 that after all corrections have been made, the intensity as measured at $67 \frac{1}{2}^{\circ}$ to the vertical with no lead absorber is from 30 to 50 percent larger than that measured in the vertical direction under the same mass of air. The effects of scattering at the other angles seem to be small and we conclude that the reduction of the values measured at the vertical because of scattering is small and probably less than 10 percent. Rossi ${ }^{15}$ estimates the effect of scattering in the initial process, when the primary forms its first secondaries, is no larger than 10 percent in reducing the intensity at the vertical. The combined effects then will probably be less than 20 percent.

We conclude then that except for large angles the intensity at an angle to the zenith at these altitudes may be predicted from the intensity at the vertical whenever the intensity at the top of the atmosphere is the same, or when the absorption in the atmosphere gives the same effective radiation in the two cases.

\section{E. East-West Effect}

The discovery of the fact that near the magnetic equator the intensity of the secondary cosmic-ray particles is greater toward the west than toward the east has an important bearing on the assumed nature of the primary particles. While these early experiments

${ }^{23}$ Winckler, Stroud. and Schenck, Phys. Rev. 74, 837 (1948). of Johnson, ${ }^{24}$ Alvarez and Compton, ${ }^{25}$ Rossi, ${ }^{26}$ and others established beyond a doubt that there was a positively charged component of the primary radiation, it was not clear whether the whole or only part of the primary particles were so charged.

A measured absence of any asymmetry in the shower producing radiation together with the small asymmetry

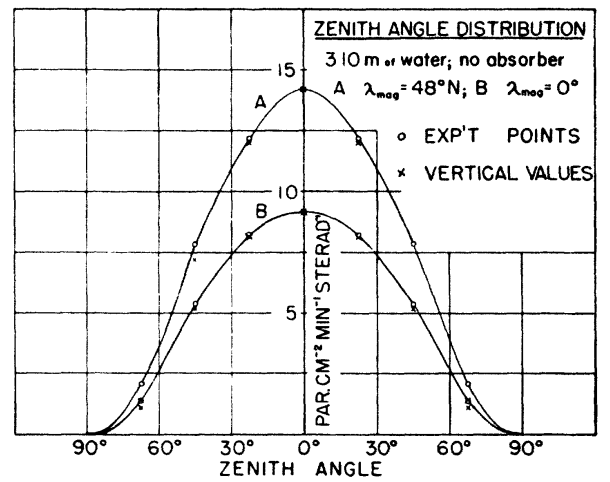

Fig. 27. Comparison of the zenith angle dependence at the equator and at $48^{\circ}$ geomagnetic North at $3.10 \mathrm{~m}$ of water with no lead absorber. Differences in values through same mass of air as at vertical are most pronounced at the larger angles and northern latitudes.

of the total radiation as measured by Johnson and Barry $^{27}$ had led these authors to the conclusion that there are two kinds of primary particles, one giving rise to the penetrating component which shows asymmetry and the other giving rise to the non-penetrating component which does not show asymmetry.

The west excess of the penetrating component has been measured by Schein, Yngre, and Kraybill ${ }^{28}$ in the geomagnetic latitude range $27^{\circ}$ to $31^{\circ}$ North at 34,500 $\mathrm{ft}$. in a B-29. They find an asymmetry of $0.46 \pm 0.07$ at a zenith angle of $45^{\circ}$ in the particles that can penetrate $22 \mathrm{~cm}$ of lead. Preliminary data on the west excess were published by the present authors ${ }^{10}$ who showed that at the highest altitude reached at the equator, corresponding to a pressure of $2.35 \mathrm{~m}$ of water, that the asymmetry in the total radiation was nearly equal to that in the penetrating. This means that there is approximately the same asymmetry in the non-penetrating as in the penetrating component at this altitude. Barber ${ }^{29}$ has reported measurements at $33,000 \mathrm{ft}$. and at $25,000 \mathrm{ft}$. near the geomagnetic equator and also concludes that there is an asymmetry in the soft as well as in the hard components.

The data here presented and shown in Figs. 12-15 and in Table IV give the measurements at different zenith angles at the equator after all corrections have

${ }^{24}$ T. H. Johnson, Phys. Rev. 43, 381 (1933).

${ }^{25}$ L. Alverez and A. H. Compton, Phys. Rev. 43, 836 (1933).

${ }^{26}$ B. Rossi, Phys. Rev. 45, 212 (1934)

${ }_{27}$ T. H. Johnson, Phys. Rev. 56, 219 (1939).

${ }^{28}$ Schein, Yngre, and Kraybill, Phys. Rev. 73, 928 (1948).

${ }^{29}$ W. C. Barber, Phys. Rev. 75, 590 (1949). 
been applied. For a continuous momentum distribution of the primary particles one would expect the curve giving the difference in intensity between the west and east to start off linearly, rise to a maximum where the atmospheric absorption becomes important and approach 0 at $90^{\circ}$. The percentage asymmetry in the intensity for large zenith angles should, as discussed by Johnson, ${ }^{30}$ decrease at large zenith angle due to the absorption of the atmosphere for particles in the momentum range responsible for the difference.

The near equality in the percentage of west excess for the penetrating and non-penetrating components again argues strongly for the common parentage for each. This, it will be recalled, is the same conclusion arrived at above for the relation between the penetrating and non-penetrating particles of the latitude sensitive part of the radiation.

For intermediate latitudes the East-West asymmetry is given by Figs. 5 and 6 . At high latitudes the difference between east and west fades to zero both without and with lead absorber. This is to be expected since no East-West effect can exist beyond the latitude where the latitude effect, in the intensity at the vertical, ceases.

\section{F. Azimuthal Effect at the Equator}

The curves given in Figs. 18 and 19 strongly suggest a sinusoidal variation with azimuth angle for a given

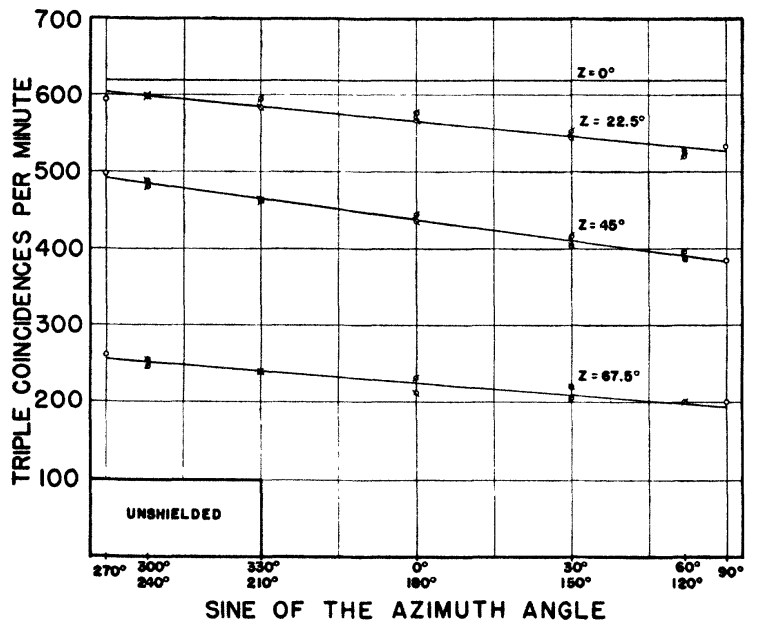

FIG. 28. Data given in Fig. 16 for no lead absorber show a sinusoidal variation with azimuth angle.

zenith angle. When plotted against the sine of the azimuth angle a straight line does in fact result within the limits of the uncertainties of the points as shown in Figs. 28 and 29.

The empirical relations that may be written down are as follows: (1) For the sine curves the number of particles per square $\mathrm{cm}$ per minute per steradian at the geomagnetic equator over Peru at $2.35 \mathrm{~m}$ of water

${ }^{30}$ T. H. Johnson, Rev. Mod. Phys. 10, 227 (1938). equivalent for the different zenith angles, $z$, may be written in terms of the azimuth angle $\varphi$ as follows:

$\begin{array}{cccc}\text { Absorber } & z=22 \frac{1}{2}^{\circ} & z=45^{\circ} & z=67 \frac{1}{2}^{\circ} \\ 0 & 10.4-0.82 \sin \varphi & 7.83-1.14 \sin \varphi & 3.12-0.60 \sin \varphi \\ 10.4 \mathrm{~cm} \mathrm{~Pb} & \cdots & 2.24-0.37 \sin \varphi & 1.16-0.18 \sin \varphi \\ 20.8 \mathrm{~cm} \mathrm{~Pb} & 2.47-0.23 \sin \varphi & 1.98-0.35 \sin \varphi & \cdots\end{array}$

The above values have been corrected for deadtime of the counters, accidentals, side showers and finite opening of the telescopes.

The ranges in momentum of the primary particles covered by these azimuth experiments, according to the analysis of Lemaitre and Vallarta are as follows (eccentricity of dipole and tilt of magnetic field taken into account) :

$\begin{array}{ccc}\text { Zenith angle } & & \\ z & P_{\min }(\mathrm{Bev} / \mathrm{c}) & P_{\max }(\mathrm{Bev} / \mathrm{c}) \\ 22 \frac{1}{2}^{\circ} & 11.2 & 17.1 \\ 45^{\circ} & 10.0 & 23.0 \\ 67 \frac{1}{2}^{\circ} & 9.3 & 32.7\end{array}$

In each complete revolution in azimuth, for each of the zenith angles covered, 12 points were taken; i.e., at every $30^{\circ}$. This means, for example, that with the unshielded telescopes, 36 ten-minute readings were taken in the momentum range 9 to $33 \mathrm{Bev} / \mathrm{c}$. It is apparent that the multiple coverage of the momentum ranges and the consistency of the behavior of the experimental points given in Figs. 18 and 19 strongly suggest a continuous momentum distribution of the primary radiation in the range from 9 to $33 \mathrm{Bev} / \mathrm{c}$. This conclusion combined with that arrived at for the latitude sensitive part means that so far as these experiments are concerned there is a continuous momentum distribution of the primary particles from 2 to $33 \mathrm{Bev} / \mathrm{c}$.

\section{G. Correlation of Latitude and East-West Effects}

It is evident, from the analysis of the behavior of charged particles in the earth's magnetic field, that the latitude effect at the vertical will be due both to

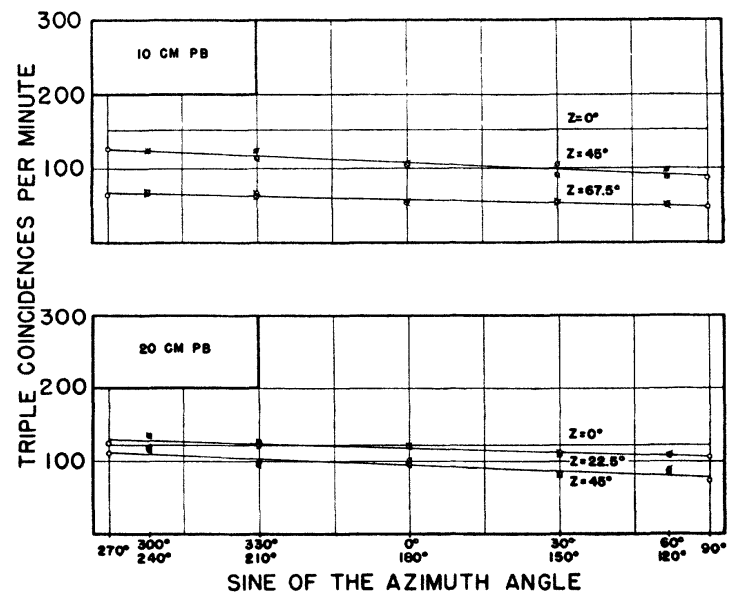

FIG. 29. Data given in Fig. 17 for 10 and $20 \mathrm{~cm}$ lead absorber show a sinusoidal variation with azimuth angle. 
positive particles and to any negatives that may exist in this momentum range. The East-West effect on the other hand is due to the difference between the numbers of positives and negatives in the particular momentum range.

Unfortunately the existing calculations on the minimum momentum allowed at different zenith angles as a function of geomagnetic latitude are not complete enough to permit an accurate check of the above. However a rough check may be made by considering the latest curves given by Vallarta. ${ }^{31}$

In accordance with the instructions, we have drawn curves through the shaded region connecting his curves $E_{1}$ and $E_{2}$ for the intermediate latitudes. As drawn we find that at zenith angle $45^{\circ}$ East the minimum momentum for positive particles at $25^{\circ}$ North geomagnetic latitude is the same as at the vertical on the geomagnetic equator. We also find in a similar way from his curves for $45^{\circ}$ West that the minimum momentum at geomagnetic latitude $25^{\circ}$ North is the same as at the vertical at $29^{\circ}$ geomagnetic north. Thus if all particles are positive we must expect the same ratio of east to west at $25^{\circ}$ North as the ratio in vertical intensities between 0 and $29^{\circ}$ North.** Taking the average of the values without and with lead taken from Figs. 7 and 8 we find for these ratios 0.82 for the former and 0.78 for the latter. Using the lead curves only where scattering is less pronounced we find for these ratios 0.80 and 0.78 respectively.

While these ratios are close, there is an uncertainty in just how the correct curves in Vallarta's figures should be drawn. It is evident, however, that no negative particles are necessary to correlate the eastwest effect and the latitude effect in this momentum range.

While the above argument eliminates the presence of an appreciable number of negative particles in the primary radiation in the momentum range 3-13 Bev/c and the near equality in the East-West effect for the non-penetrating and penetrating particles argues for a common positive particle responsible for this difference in the momentum range 9 to $33 \mathrm{Bev} / \mathrm{c}$, it should be pointed out that these experiments do not mean that there is but one kind of positive particle in the primary radiation. While it seems most likely that the major part of the momentum is brought in at low latitudes by protons, the presence of some $\alpha$ - or heavier particles cannot be excluded. The relative numbers of such particles, however, near the equator must be small in view of the constant proportionality at all altitudes between a single counter and an ionization chamber even up to $80,000 \mathrm{ft}^{32}$

\footnotetext{
${ }^{31}$ M. S. Vallarta, Phys. Rev. 74, 1838 (1948).

** There might be some uncertainty introduced because of the larger amount of air at zenith angle $45^{\circ}$ compared with the vertical. We find, however, from Fig. 22, that the latitude effect at 3.10 (secant $45^{\circ}$ ) $\mathrm{m}$ of water is very closely the same as at $3.10 \mathrm{~m}$ of water.

${ }^{32}$ H. V. Neher and W. H. Pickering, Phys. Rev. 61, 409 (1942).
}

\section{H. The Latitude Effect of the Total Radiation}

The latitude effect of the total radiation as measured with the ionization chamber and shown in Fig. 9 is 36.1 percent as compared with that measured with the vertically directed unshielded telescopes of 37.0 percent. An agreement is to be expected if (1) the zenith angle distribution at this altitude does not change between the northern latitudes and the equator and (2) the relative numbers of the different kinds of particles do not change. The asymmetry at the equator will, to a close approximation, be unimportant in this comparison.

Actually it will be seen from Fig. 27 that the zenith angle distribution is somewhat flatter at the equator than at $48^{\circ}$ North. The integrated effect over all zenith angles gives a ratio of total to vertical about 4 percent larger at the equator than at the northern latitude. This result combined with the slightly smaller latitude effect with the ionization chamber indicates that the average ionization per particle at the equator at this altitude is about 6 percent smaller than at $48^{\circ}$ North.

\section{Upward Radiation}

The question as to how much upward radiation is present at the altitudes reached here has often been raised. Some data that bears on this question can be given from the experiments performed during these flights. On several occasions the two East-West frameworks were turned vertical. In one of these frameworks a threefold coincidence telescope was shielded from below by $10 \mathrm{~cm}$ of lead and in the other by $20 \mathrm{~cm}$ of lead. In each there was no absorbing material except the counter walls and the skin of the plane for the radiation coming in the downward direction. The counting rates of these telescopes could then be compared with the two telescopes which were used to measure the total radiation and had no absorber, either top or bottom. The ratio of the counting rates of the telescope shielded below with $10 \mathrm{~cm}$ of lead to the average of the unshielded ones at $30,000 \mathrm{ft}$. was 0.998 . At Inyokern on the ground the ratio was 1.004. For the telescope shielded from below with $20 \mathrm{~cm}$ of lead the corresponding ratios were 0.989 and 0.983 .

This agreement between relative response of telescopes with no lead below and with 10 and $20 \mathrm{~cm}$ of lead below means (1) that the amount of upward radiation through the unshielded telescopes at these altitudes was negligibly small, and (2) that the number of events occurring in the lead such as showers, nuclear explosions, etc., that send particles upward through the telescopes that had lead below was negligibly small.

\section{J. Production of Penetrating Particles in the Atmosphere}

Experimental data near sea level agree with theoretical calculations for the absorption of cosmic-ray mesons in various materials. In particular Ross ${ }^{15}$ states that a lead thickness of $167 \mathrm{~g} \mathrm{~cm}^{-2}$ corresponds to an 
air thickness of $100 \mathrm{~g} \mathrm{~cm}^{-2}$ as far as total energy losses of mesons due to collisions are concerned. The absorbing layers used in these experiments including the iron box for holding the lead were 10.4 and 20.8 equivalent $\mathrm{cm}$ of lead. This should correspond in absorption on the above basis to 71 and $142 \mathrm{~g} \mathrm{~cm}^{-2}$ of air respectively.

Actually in the atmosphere, decay of the mesons plays an important part in the apparent absorption of these particles. ${ }^{33,34}$ Using the results of Rossi, Hilberry, and Hoag, and assuming the same average momentum of the mesons at $2.35 \mathrm{~m}$ of water equivalent at the equator as at Mt. Evans we find that by changing altitude by an amount which adds $55 \mathrm{~g} \mathrm{~cm}^{-2}$ of air should be the equivalent of adding the second $10.4 \mathrm{~cm}$ of lead. Actually it is seen from the lead curves given in Fig. 21 that it requires an addition of $85 \mathrm{~g} \mathrm{~cm}^{-2}$ of air at $2.35 \mathrm{~m}$ of water at the equator to drop the counting rate with the $10.4 \mathrm{~cm}$ of lead down to that obtained with $20.8 \mathrm{~cm}$ of lead at the high elevation. The same situation exists for corresponding data at $50^{\circ}$ North but here the lead curves are not as accurately determined as at the equator.

The possible interpretations of this behavior are: (1) Due to different kinds of particles at these high altitudes the absorption is different than at sea level. (2) There is production of penetrating particles taking place in the atmosphere between the two levels. This production may be taking place in the lead as well but will not show since the lead is between the counters and not above.

In terms of the total number of penetrating particles at $2.35 \mathrm{~m}$ of water at the equator the cross section for production of new penetrating particles is $1.0 \times 10^{-25}$ $\mathrm{cm}^{2}$. In terms of Rossi's N-rays ${ }^{15}$ the cross section is about $4 \times 10^{-25} \mathrm{~cm}^{2}$. This latter number is about equal to the cross section usually taken for the nitrogen nucleus.

It seems quite likely that production of mesons is taking place in the atmosphere at these altitudes and in fact at the equator one might expect the lead curves to reach a maximum at from 1 to $2 \mathrm{~m}$ of water equivalent. At this point the rate of production would be just equal to the rate of absorption.

The argument for the lead curves passing through a maximum at the equator while they apparently do not at $50^{\circ}$ geomagnetic North $^{35}$ is as follows: We take the areas under the ionization curves as a measure of the total energy being brought in by the primary particles. The data of Bowen, Millikan and Neher $^{36}$ combined with an assumed differential numbers distribution of $p^{-2.5}$ for the particles remaining at the equator show that there are incident on the top of the atmosphere at the equator only one-eighth the number at $50^{\circ}$ North.

\footnotetext{
${ }^{33}$ Rossi, Hilberry, and Hoag, Phys. Rev. 57, 461 (1940).

${ }^{34}$ H. V. Neher and H. G. Stever, Phys. Rev. 58, 766 (1940).

${ }_{35}$ M. A. Pomerantz, Phys. Rev. 75, 69 (1949)

${ }^{36}$ Bowen, Millikan, and Neher, Phys. Rev. 53, 859 (1938).
}

However the lead curves at one-third atmosphere at the equator are only 35 percent less than at $50^{\circ}$ North. We thus infer that had we taken our vertical telescopes containing lead up to very high altitudes at the equator we would have measured a value considerably lower than at our highest elevation ( $2.35 \mathrm{~m}$ of water). If $4 \times 10^{-25} \mathrm{~cm}^{2}$ is taken for the cross section for interaction of the primary particles with the air nuclei then the maximum might be expected at 1 to $2 \mathrm{~m}$ of water equivalent air pressure.

\section{K. Latitude Effect of Wide Showers}

The data given in Fig. 10 indicate that within the rather appreciable probable errors given there is no latitude effect in the extensive or wide showers. This is interpreted as meaning that the primary particles responsible for these large showers are either electrically neutral or have sufficient momenta as to be unaffected by the earth's magnetic field.

\section{Altitude Dependence of Wide Showers}

Kraybill $^{37}$ has reported a maximum at about $3.5 \mathrm{~m}$ of water in the counting rate versus pressure of the atmosphere curve for extended atmospheric showers at northern latitudes. The data shown in Fig. 11 where the point taken at $2.35 \mathrm{~m}$ of water was at the magnetic equator confirms the result of Kraybill in placing the maximum at about one-third atmosphere. Since there appears to be no latitude dependence for wide showers one would expect the same altitude dependence at all latitudes.

The increase from the ground level at Inyokern (9.60 $\mathrm{m}$ of water) to the peak of the curve is a factor of 33. Counter telescope data show that the particles that cannot penetrate $10 \mathrm{~cm}$ of lead increase by a factor of 40 in going from the altitude of Inyokern to $3.1 \mathrm{~m}$ of water. This is also the rate of increase of shower producing radiation and also of neutrons in the lower part of the atmosphere.

It is probably no accident that the absorption of extended shower particles at depths in the atmosphere beyond the peak of the curve is close to the same as that for the shower producing radiation. An initiating, very high energy electron or photon, near the top of the atmosphere would produce a large cascade, which in our case builds up to its maximum at $3.5 \mathrm{~m}$ of water. Beyond the peak, shower theory shows that the average energy of the particles is nearly independent of the energy of the initiating particle and that the absorption thickness, i.e., the thickness for the number of ionizing particles to drop to $1 / e$ of their initial value, is approximately $150 \mathrm{~g} \mathrm{~cm}^{-2}$. This is just the value found above for the absorption of the extensive showers at some distance beyond the peak of the curve. The presence of a small percentage of penetrating particles accompanying these extensive showers, such as are known to

\footnotetext{
${ }^{37}$ H. L. Kraybill, Phys. Rev. 73, 632 (1948).
} 
exist, will not appreciably change the main features of the absorption curve.

\section{SUMMARY}

The cosmic-ray experiments herein reported were made in a B-29 flying along $80^{\circ}$ West longitude at a pressure altitude of $30,000 \mathrm{ft}$. from $54^{\circ}$ North to the geomagnetic equator during a two-week period in June of 1948. The fact that the results of the return trip from Peru agree with those taken going down indicates that no significant changes in the primary cosmic-ray intensity took place during this period. In spite of the 300 -m.p.h. speed of the plane the counting rates of the Geiger counter telescopes were such that the probable error of no point on the latitude effect curves is more than 2 percent, even with $20 \mathrm{~cm}$ of lead absorber. To achieve this accuracy and at the same time keep a relatively small aperture, large area counter trays were used.

From the data given the following conclusions are drawn: (1) There is the same ratio between penetrating and non-penetrating radiation at $3.10 \mathrm{~m}$ of water equivalent at the equator as at northern latitudes, i.e., there is the same latitude effect for each. This same ratio of penetrating to non-penetrating is found at the equator for the particles causing the east-west difference. It is also found for the radiation coming in at $45^{\circ}$ East when the mass of air penetrated is the same. From these findings we conclude that whatever the process by which the primary particles are transformed into secondaries, the relative number of penetrating and non-penetrating particles appearing after traversing one-third atmosphere of air is the same irrespective of the momentum of the primary particle. The fact that this conclusion holds also for the radiation remaining at the equator argues for these particles being the same as those showing geomagnetic effects. (2) The present data permits us to redraw our older count versus depth curves, normalizing them all to the present latitude curve at $3.10 \mathrm{~m}$ of water. After the adjustment we find no cut-off of low energy particles up to $60^{\circ}$ geomagnetic north. This corresponds to a minimum momentum at the vertical of about $1 \mathrm{Bev} / \mathrm{c}$. (3) From the azimuthal experiments made at $2.35 \mathrm{~m}$ of water over Peru we find a smooth variation in azimuth for all zenith angles, namely $22 \frac{1}{2}^{\circ}, 45^{\circ}$ and $67 \frac{1}{2}^{\circ}$. These measurements sweep across a momentum range of the primary particles (according to Vallarta et al.) from 9 to $33 \mathrm{Bev} / \mathrm{c}$. This fact, taken together with the smooth variation with latitude found for the vertical radiation as well as for that coming in at $45^{\circ}$ East and $45^{\circ}$ West, means that, in the momentum range from about 2 to $33 \mathrm{Bev} / \mathrm{c}$, as far as these experiments are concerned, there is a continuous momentum distribution of the particles whose effects can reach down to where these experiments were performed. (4) Comparison of the EastWest effect and latitude effect, in a region where particles of the same momentum range (according to Vallarta) are involved, shows that the two are equal. This can only be so if the number of negative particles in the primary radiation, whose effects reach through one-third atmosphere, is negligible compared with the positives, in this momentum range. (5) Evidence for the production of penetrating particles at these altitudes is found when the absorption in air and lead is compared. If Rossi's "N-rays" are responsible for this production the cross section is about equal to that of the nitrogen nucleus. (6) Measurements on extended showers indicate that they are not latitude sensitive. They may thus be due either to electrically neutral primaries or, what seems to be more probable, to charged particles whose momentum is so great as to be unaffected by the earth's magnetic field. (7) The altitude dependence of extended showers shows a maximum at about one-third atmosphere. Beyond the maximum they show an absorption in the atmosphere about equal to that of either the soft component or the shower producing component. (8) The latitude effect of the total radiation as measured with an ionization chamber shows very closely the same latitude effect at $3.10 \mathrm{~m}$ of water as the vertical counter telescopes. This is to be expected if (a) the zenith angle effect is not latitude sensitive and (b) the relative numbers of the different kinds of particles that ionize differently at this altitude does not change appreciably with latitude. The slightly different zenith angle dependence found, together with the small difference in the latitude effect indicates an average ionization per particle at the equator slightly less than at the northern latitudes. (9) The amount of upward radiation that can be stopped by $20 \mathrm{~cm}$ of lead is negligible at $3.10 \mathrm{~m}$ of water. (10) The amount of backward scattering together with nuclear events in the lead, that result in particles ejected upward which can pass through a telescope mounted over the lead, are negligible compared with the downward radiation.

In conclusion the authors wish to thank the Office of Naval Research and the United States Air Forces for making these flights possible. Above all we wish to express our appreciation to Major W. A. Gustafson, Captain Freyer, Lt. Brandon and the other members of the crew of the B-29 for their cooperation and efforts spent in making these flights successful. 